\title{
SPECIALIZATION AND PICARD-VESSIOT THEORY
}

\author{
BY \\ LAWRENCE GOLDMAN
}

Introduction. Let $\mathcal{G}$ be a field and let $t, \bar{t}$ be elements of some extension field of $g$. One says that $t \rightarrow \bar{t}$ is a specialization over $g$ if for every polynomial $F(x) \in \mathcal{G}[x]$ such that $F(t)=0$ we have $F(\bar{t})=0$. Let $F(t, x)=a_{0}(t) x^{n}+\cdots$ $+a_{n}(t) \in \mathcal{g}[t, x]$ be an irreducible polynomial in $x$ over $\mathcal{G}(t)$ and let $t \rightarrow \bar{t}$ be a specialization over $\mathcal{G}$ such that $a_{0}(t) d(\bar{t}) \neq 0$, where $d(t)$ is the discriminant of $F$, then the specialization $t \rightarrow \bar{t}$ over $\mathcal{G}$ can be extended to a specialization $\left(t, x_{1}, \cdots, x_{n}\right) \rightarrow\left(\bar{t}, \bar{x}_{1}, \cdots, \bar{x}_{n}\right)$ over $\mathcal{G}$ where $\left(x_{1}, \cdots, x_{n}\right),\left(\bar{x}_{1}, \cdots, \bar{x}_{n}\right)$ are the roots of $F(t, x), F(\bar{t}, x)$ respectively. Furthermore, the group $H$ of automorphisms of $\mathcal{G}\left(\bar{t}, \bar{x}_{1}, \cdots, \bar{x}_{n}\right)$ over $\mathcal{G}(\bar{t})$, considered as a permutation group on $1,2, \cdots, n$, is a subgroup of the group $G$ of automorphisms of $\mathcal{G}\left(t, x_{1}, \cdots, x_{n}\right)$ over $\mathcal{G}(t)$, also considered as a permutation group on $1,2, \cdots, n$ (van der Waerden [5]).

The purpose of part I of this paper is to obtain analogous results for homogeneous linear ordinary differential polynomials.

Let $\mathcal{F}$ be an ordinary differential field of characteristic zero (i.e., a field of characteristic zero with a given derivation) whose field of constants $C$ is algebraically closed. Let $t_{1}, \cdots, t_{r}, \bar{t}_{1}, \cdots, \bar{t}_{r}$ be elements of some differential field extension of $\mathcal{F}$; then $\left(t_{1}, \cdots, t_{r}\right) \rightarrow\left(\bar{t}_{1}, \cdots, \bar{t}_{r}\right)$ is a specialization over $\mathcal{F}$ if for any differential polynomial $F\left(y_{1}, \cdots, y_{r}\right) \in \mathcal{F}\left\{y_{1}, \cdots, y_{r}\right\}$ such that $F\left(t_{1}, \cdots, t_{r}\right)=0$ we have $F\left(\bar{t}_{1}, \cdots, \bar{t}_{r}\right)=0$. The specialization $\left(t_{1}, \cdots, t_{r}\right)$ $\rightarrow\left(\bar{t}_{1}, \cdots, \bar{t}_{r}\right)$ over $\mathcal{F}$ is generic if $\left(\bar{t}_{1}, \cdots, \bar{t}_{r}\right) \rightarrow\left(t_{1}, \cdots, t_{r}\right)$ is also a specialization over $\mathcal{F}$. If $\mathcal{G}$ is a differential field extension of $\mathcal{F}$ and $\beta$ is a constant transcendental over $\mathcal{G}$ we may form the differential field $g((\beta))$ of all formal power series in $\beta$ with coefficients in $\mathcal{G}$ and only a finite number of terms with negative exponents. Let $f=f_{0}+\sum_{i=1}^{\infty} f_{i} \beta^{i} \in \mathcal{G}((\beta))$ and let $f$ be a zero of $F(x)$ $\in \mathcal{F}\{x\}$; then $F\left(f_{0}\right)=0$, because $F\left(f_{0}\right)$ is the term of $F(f)$ of degree 0 in $\beta$, so that $f \rightarrow f_{0}$ is a specialization over $\mathcal{F}$. We call a specialization $\left(t_{1}, \cdots, t_{r}\right)$ $\rightarrow\left(\bar{t}_{1}, \cdots, \bar{t}_{r}\right)$ over $\mathcal{F}$ analytic if there exist $r$ elements $\bar{t}_{i}+\sum_{j=1}^{\infty} f_{i j} \beta^{i} \in \mathcal{G}((\beta))$ $(i=1, \cdots, r)$, where $\mathcal{G}$ is some differential field extension of $\mathcal{F}$, such that $\left(t_{1}, \cdots, t_{r}\right) \rightarrow\left(\bar{t}_{1}+\sum_{j} f_{1 j} \beta^{j}, \cdots, \bar{t}_{r}+\sum f_{r j} \beta^{j}\right)$ is a generic specialization over F.

Corollary 2 of Lemma 2 shows that if $\bar{t}$ is not a singular solution of $F(y)$ $=0$, where $F(y)$ is the irreducible differential polynomial in $\mathcal{F}\{y\}$ of lowest order vanishing at $t$, then the specialization $t \rightarrow \bar{t}$ over $\mathcal{F}$ is analytic.

Let $L(t, y)=a_{0}(t) y^{(n)}+\cdots+a_{n}(t) y \in \mathcal{F}\{t, y\}$, where $t$ denotes $\left(t_{1}, \cdots, t_{r}\right)$, let $t \rightarrow \bar{t}$ be an analytic specialization over $\mathcal{F}$ such that $a_{0}(\bar{t}) \neq 0$ and let

Received by the editors May 15, 1956. 
$\left(\lambda_{1}, \cdots, \lambda_{n}\right)$ be a fundamental set of zeros of $L(\bar{t}, y)$; then Theorem 1 states that there exists a fundamental system of zeros $\left(\omega_{1}, \cdots, \omega_{n}\right)$ of $L(t, y)$ such that $\left(t, \omega_{1}, \cdots, \omega_{n}\right) \rightarrow\left(\bar{t}, \lambda_{1}, \cdots, \lambda_{n}\right)$ is an analytic specialization over $\mathcal{F}$.

If $G$ is a differential field with an algebraically closed field of constants $D$ then $\mathcal{G}\left\langle\omega_{1}, \cdots, \omega_{n}\right\rangle$ is called a Picard-Vessiot extension (hereafter denoted by P.V.E.) of $g$ if the field of constants of $g\left\langle\omega_{1}, \cdots, \omega_{n}\right\rangle$ is $D$ and $\left(\omega_{1}, \cdots, \omega_{n}\right)$ is a fundamental system of zeros of a homogeneous linear differential polynomial of order $n$ (Kolchin [2]). Note that Theorem 1 does not say anything about the field of constants of $\mathcal{F}\left\langle t, \omega_{1}, \cdots, \omega_{n}\right\rangle$. In fact, as we shall show by examples, $\mathcal{F}\left\langle t, \omega_{1}, \cdots, \omega_{n}\right\rangle$ may not be a P.V.E. of $\mathcal{F}\langle t\rangle$ even when the field of constants of $\mathscr{F}\langle t\rangle$ is algebraically closed.

Let $\mathcal{G}$ be a differential field extension of $\mathcal{F}$ and let the field of constants of $\mathcal{F}$ and $\mathcal{G}$ be $C$ which is algebraically closed. Let $t \rightarrow \bar{t}+\sum_{i=1}^{\infty} f_{i} \beta^{i} \in \mathcal{G}((\beta))$ be a generic specialization over $\mathcal{F}$. Let $E$ be an algebraic closure of the field $C((\beta))$ and let $\left(\omega_{1}, \cdots, \omega_{n}\right),\left(\lambda_{1}, \cdots, \lambda_{n}\right)$ be fundamental systems of zeros of $L(t, y), L(\bar{t}, y)$ respectively as given by Theorem 1 . Under these conditions Theorem 2 states:

(1) $\mathcal{F}\left\langle t, \omega_{1}, \cdots, \omega_{n}, E\right\rangle$ is a P.V.E. of $\mathcal{F}\langle t, E\rangle$.

(2) If $G^{E}$ respectively $H^{C}$ is the group of all automorphisms of $\mathcal{F}\left\langle t, \omega_{1}, \cdots, \omega_{n}, E\right\rangle$ over $\mathfrak{F}\langle t, E\rangle$ respectively $\mathcal{G}\left\langle\lambda_{1}, \cdots, \lambda_{n}\right\rangle$ over $\mathcal{G}$ (identified with an algebraic matric group with coefficients in $E$ respectively $C$ by the given fundamental system of zeros $\left(\omega_{1}, \cdots, \omega_{n}\right)$ respectively $\left.\left(\lambda_{1}, \cdots, \lambda_{n}\right)\right)$, then the analytic specialization $\left(t, \omega_{1}, \cdots, \omega_{n}\right) \rightarrow\left(\bar{t}, \lambda_{1}, \cdots, \lambda_{n}\right)$ over $\mathcal{F}$ induces an analytic specialization of the elements of a certain subgroup $K^{E}$ of $G^{E}$ which is a group homomorphism of $K^{E}$ onto $H^{C}$. In particular if the field of constants of $\mathscr{F}\left\langle t, \omega_{1}, \cdots, \omega_{n}\right\rangle$ is $C$ then $H^{C}$ is a subgroup of $G^{C}$.

Theorems 3, 4, and 5 give sufficient conditions for the existence of an extension of an analytic specialization $t \rightarrow \bar{t}$ over $\mathcal{F}$ to a specialization $\left(t, \omega_{1}, \cdots, \omega_{n}\right) \rightarrow\left(\bar{t}, \lambda_{1}, \cdots, \lambda_{n}\right)$ over $\mathcal{F}$ where $\mathcal{F}\left\langle t, \omega_{1}, \cdots, \omega_{n}\right\rangle$ is a P.V.E. of $\mathcal{F}\langle t\rangle$, under the added assumption that the field of constants of $\mathcal{F}\langle t, \bar{t}\rangle$ is the same as that of $\mathcal{F}$, namely $C$.

In part II we introduce the notion of a "generic equation with group $G$ " for homogeneous linear differential equations of order $n$. This is analogous to what E. Noether did for algebraic equations (E. Noether [4]). Roughly speaking, given an $n \times n$ algebraic matric group $G$ we seek an $n$th order homogeneous linear differential polynomial $L(t, y) \in C\left\langle t_{1}, \cdots, t_{n}\right\rangle\{y\}$, where $t=\left(t_{1}, \cdots, t_{n}\right)$ is a family of $n$ differential indeterminates over $C$ such that there exists a fundamental system of zeros $\left(y_{1}, \cdots, y_{n}\right)$ of $L(t, y)$ with the following properties:

(1) $C\left\langle y_{1}, \cdots, y_{n}\right\rangle$ is a P.V.E. of $C\left\langle t_{1}, \cdots, t_{n}\right\rangle$ with group of automorphisms $G$.

(2) For any specialization $\left(t_{1}, \cdots, t_{n}\right) \rightarrow\left(\bar{t}_{1}, \cdots, \bar{t}_{n}\right)$ over $C$ which can be extended to a specialization $\left(t_{1}, \cdots, t_{n}, y_{1}, \cdots, y_{n}\right) \rightarrow\left(\bar{t}_{1}, \cdots, \bar{t}_{n}\right.$, 
$\left.\bar{y}_{1}, \cdots, \bar{y}_{n}\right)$ with $C\left\langle\bar{t}_{1}, \cdots, \bar{t}_{n}, \bar{y}_{1}, \cdots, \bar{y}_{n}\right\rangle$ a P.V.E. of $C\left\langle\bar{t}_{1}, \cdots, \bar{t}_{n}\right\rangle$ the algebraic matric group $H$ of $C\left\langle\bar{t}_{1}, \cdots, \bar{t}_{n}, \bar{y}_{1}, \cdots, \bar{y}_{n}\right\rangle$ over $C\left\langle\bar{t}_{1}, \cdots, \bar{t}_{n}\right\rangle$ is a subgroup of $G$.

(3) If $\mathcal{F}$ is a differential field with field of constants $C$ and if $\mathcal{F}\left\langle\lambda_{1}, \cdots, \lambda_{n}\right\rangle$ is a P.V.E. of $\mathcal{F}$ with group $H \subseteq G$, where $\left(\lambda_{1}, \cdots, \lambda_{n}\right)$ is a fundamental system of zeros of a homogeneous linear differential polynomial $L(y) \in \mathcal{F}\{y\}$ of order $n$, there exists a specialization $\left(t_{1}, \cdots, t_{n}\right) \rightarrow\left(\bar{t}_{1}, \cdots, \bar{t}_{n}\right)$ over $C$ such that $\bar{t}_{i} \in \mathcal{F}(i=1, \cdots, n)$ and $L(\bar{t}, y)=L(y)$.

By an argument similar to that which E. Noether used, we show that the existence of a "generic equation with group $G$ " implies that the differential subfield of $C\left\langle y_{1}, \cdots, y_{n}\right\rangle$ consisting of the invariants of $G$ is purely differentially transcendental over $C$. We then proceed to show how to construct a "generic equation with group $G$ " of any order $n$ for the following groups $G$ :

(1) Full linear group.

(2) Unimodular group.

(3) Reducible group consisting of all nonsingular matrices $\left(a_{i j}\right)(i, j$ $=1, \cdots, n)$ such that $a_{r+k, m}=0(k=1, \cdots, s ; m=1, \cdots, r ; r+s=n)$.

(4) Orthogonal group.

(5) Symplectic group.

I wish to take this opportunity to thank Professor Ellis R. Kolchin for the numerous valuable suggestions and criticisms that he has given me, without which this paper would not have been possible.

Notation. Throughout this paper $\mathfrak{F}$ will stand for an ordinary differential field of characteristic zero whose field of constants $C$ is algebraically closed. We shall use $B, D, E$ for fields of constants which contain $C$. $G, H$ will denote algebraic matric groups with coefficients in $C ; G^{E}, H^{E}$ will stand for algebraic matric groups with coefficients in $E$. $[F]$ means the differential ideal generated by $F,\{F\}$ means the perfect (radical) differential ideal generated by $F$, in some specified differential ring. By the separant of a differential polynomial $F(y)$ in an indeterminate $y$ we mean $\partial F / \partial y^{(r)}$ where $r$ is the order of $F$. $t_{1}, \cdots, t_{r}$ will always denote elements of a differential field extension of $\mathcal{F}$; the point $\left(t_{1}, \cdots, t_{r}\right)$ will frequently be denoted by $t . W\left(y_{1}, \cdots, y_{r}\right)$ will always stand for the Wronskian of $y_{1}, \cdots, y_{r}$.

\section{Specializations AND P.v.E.}

\section{Fundamental systems of zeros.}

Lemma 1. Let $\left(\omega_{1}, \cdots, \omega_{n}\right)$ be a fundamental system of zeros of a homogeneous linear differential polynomial $L(y) \in \mathcal{F}\{y\}$ of order $n$. Let the field of constants of $\mathcal{F}\left\langle\omega_{1}, \cdots, \omega_{n}\right\rangle$ be $D \supseteq C$ and let $\bar{D}$ be the algebraic closure of $D$. Then there exists a fundamental system of zeros $\mu_{i}=\sum_{j=1}^{n} a_{i j} \omega_{j}(i=1, \cdots, n)$ of $L(y)$ such that $\mathscr{F}\left\langle\mu_{1}, \cdots, \mu_{n}\right\rangle$ is a P.V.E. of $\mathcal{F}$ and $a_{i j} \in \bar{D}(i, j=1, \cdots, n)$.

Proof. Of all fundamental systems of zeros of $L(y)$ let $\left(\pi_{1}, \cdots, \pi_{n}\right)$ be 
one such that degree of transcendency of $\mathcal{F}\left\langle\pi_{1}, \cdots, \pi_{n}\right\rangle$ over $\mathcal{F}$ is as small as possible. By Kolchin's existence theorem (Kolchin [1]) $\mathcal{F}\left\langle\pi_{1}, \cdots, \pi_{n}\right\rangle$ is a P.V.E. of $\mathcal{F}$. Also, $\pi_{i}=\sum_{j=1}^{n} b_{i j} \omega_{j}$ where each $b_{i j}$ is a constant. There, obviously, exists a specialization $\left(b_{i j}\right) \rightarrow\left(a_{i j}\right)$ over $\mathscr{F}\left\langle\omega_{1}, \cdots, \omega_{n}\right\rangle$ with each $a_{i j} \in \bar{D}$ such that determinant $\left(a_{i j}\right) \neq 0$. Let $\mu_{i}=\sum_{j=1}^{n} a_{i j} \omega_{j}$; then any differential polynomial $P \in \mathcal{F}\left\{y_{1}, \cdots, y_{n}\right\}$ which vanishes at $\left(\pi_{1}, \cdots, \pi_{n}\right)$ will vanish at $\left(\mu_{1}, \cdots, \mu_{n}\right)$, so that $\left(\mu_{1}, \cdots, \mu_{n}\right)$ is a specialization of $\left(\pi_{1}, \cdots, \pi_{n}\right)$ over $\mathcal{F}$. Hence the transcendence degree of $\mathcal{F}\left\langle\mu_{1}, \cdots, \mu_{n}\right\rangle$ over $\mathcal{F}$ is $\leqq$ that of $\mathcal{F}\left\langle\pi_{1}, \cdots, \pi_{n}\right\rangle$; since the latter is minimal, the two transcendence degrees are equal, so that $\left(\mu_{1}, \cdots, \mu_{n}\right)$ is a generic specialization of $\left(\pi_{1}, \cdots, \pi_{n}\right)$ over $\mathcal{F}$. Hence $\mathcal{F}\left\langle\mu_{1}, \cdots, \mu_{n}\right\rangle$ is a P.V.E. of $\mathcal{F}$ and $\mu_{i}=\sum a_{i j} \omega_{j}\left(a_{i j} \in \bar{D}\right)$.

Corollary 1. Let $L(y) \in \mathfrak{F}\{y\}$ be a homogeneous linear differential polynomial of order $n$. Let $\left(\omega_{1}, \cdots, \omega_{n}\right)$ and $\left(\pi_{1}, \cdots, \pi_{n}\right)$ be two fundamental systems of zeros of $L(y)$ each generating a P.V.E. of $\mathcal{F}$ and let $G$ and $H$ be their respective groups, each identified with an algebraic matric group by the respective fundamental system. Then there exists an isomorphism of $\mathcal{F}\left\langle\omega_{1}, \cdots, \omega_{n}\right\rangle$ onto $\mathcal{F}\left\langle\pi_{1}, \cdots, \pi_{n}\right\rangle$ over $\mathcal{F}$ and there exists an invertible $n \times n$ matrix $S$ over $C$ such that $H=S G S^{-1}$.

Proof. Let $\left(\mu_{1}, \cdots, \mu_{n}\right)$ be a fundamental system of zeros of $L(y)$ with degree of transcendency of $\mathcal{F}\left\langle\mu_{1}, \cdots, \mu_{n}\right\rangle$ over $\mathcal{F}$ as small as possible. Let $\mu_{i}=\sum_{j=1}^{n} b_{i j} \omega_{j}(i=1, \cdots, n)$. Then as in the proof of Lemma 1 there exists a generic specialization $\left(\lambda_{1}, \cdots, \lambda_{n}\right)$ of $\left(\mu_{1}, \cdots, \mu_{n}\right)$ over $\mathcal{F}$ such that $\lambda_{i}=\sum a_{i j} \omega_{j}(i=1, \cdots, n)$ with each $a_{i j} \in C$, so that $\mathscr{F}\left\langle\omega_{1}, \cdots, \omega_{n}\right\rangle$ $=\mathscr{F}\left\langle\lambda_{1}, \cdots, \lambda_{n}\right\rangle$ and the matric group of $\mathscr{F}\left\langle\lambda_{1}, \cdots, \lambda_{n}\right\rangle$ over $\mathcal{F}$ is $T^{-1} G T$ where $T=\left(a_{i j}\right)$. Since $\left(\lambda_{1}, \cdots, \lambda_{n}\right)$ is a generic specialization of $\left(\mu_{1}, \cdots, \mu_{n}\right)$ over $\mathcal{F}, \mathcal{F}\left\langle\mu_{1}, \cdots, \mu_{n}\right\rangle$ is isomorphic to $\mathcal{F}\left\langle\lambda_{1}, \cdots, \lambda_{n}\right\rangle=\mathscr{F}\left\langle\omega_{1}, \cdots, \omega_{n}\right\rangle$ and the group of $\mathcal{F}\left\langle\mu_{1}, \cdots, \mu_{n}\right\rangle$ over $\mathcal{F}$ is also $T^{-1} G T$. By the same argument $\mathcal{F}\left\langle\pi_{1}, \cdots, \pi_{n}\right\rangle$ is isomorphic to $F\left\langle\mu_{1}, \cdots, \mu_{n}\right\rangle$ and the group of $F\left\langle\mu_{1}, \cdots, \mu_{n}\right\rangle$ is similar to $H$. Hence $\mathscr{F}\left\langle\pi_{1}, \cdots, \pi_{n}\right\rangle$ is isomorphic to $\mathscr{F}\left\langle\omega_{1}, \cdots, \omega_{n}\right\rangle$ and $H$ is similar to $G$, i.e., is of the form $S G S^{-1}$.

COROLlary 2. Let $\left(\omega_{1}, \cdots, \omega_{n}\right)$ be a fundamental system of zeros of a homo. geneous linear differential polynomial $L(y) \in \mathcal{F}\{y\}$ of order $n$. Let the field of constants of $\mathcal{F}\left\langle\omega_{1}, \cdots, \omega_{n}\right\rangle$ be $D \supseteq C$. Let $\bar{D}$ be the algebraic closure of $D$. Let $\pi_{i}=\sum_{j=1}^{n} \dot{b}_{i j} \omega_{j}(i=1, \cdots, n)$ be a fundamental system of zeros of $L(y)$ such that $\mathfrak{F}\left\langle\pi_{1}, \cdots, \pi_{n}\right\rangle$ is a P.V.E. of $\mathcal{F}$. Then there exists a generic specialization $\left(\pi_{1}, \cdots, \pi_{n}\right) \rightarrow\left(\mu_{1}, \cdots, \mu_{n}\right)$ over $\mathcal{F}$ where $\mu_{i}=\sum_{j=1}^{n} a_{i j} \omega_{j}$ with each $a_{i j} \in \bar{D}$.

Proof. By Corollary 1 the transcendence degree of all P.V.E. of $\mathfrak{F}$ associated with $L(y)$ over $\mathcal{F}$ are equal. Hence degree of transcendency of $\mathcal{F}\left\langle\pi_{1}, \cdots, \pi_{n}\right\rangle$ over $\mathcal{F}$ is least. Then, as in the proof of Lemma 1 , there exists a generic specialization $\left(\pi_{1}, \cdots, \pi_{n}\right) \rightarrow\left(\mu_{1}, \cdots, \mu_{n}\right)$ over $\mathcal{F}$ such that $\mu_{i}=\sum_{j=1}^{n} a_{i j} \omega_{j}$ with $a_{i j} \in \bar{D}$. 
CoROllaRy 3. Let the field of constants of $\mathcal{F}\langle s, \bar{s}\rangle$ be $C$ and let $s \rightarrow \bar{s}$ be a generic specialization over F. Let $\left(\lambda_{1}, \cdots, \lambda_{n}\right)$ be a fundamental system of zeros of $L(s, y)=a_{0}(s) y^{(n)}+\cdots+a_{n}(s) y \in \mathcal{F}\langle s\rangle\{y\}$ such that the field of constants of $\mathcal{F}\left\langle s, \bar{s}, \lambda_{1}, \cdots, \lambda_{n}\right\rangle$ is $C$. Then there exists a fundamental system of zeros $\left(\mu_{1}, \cdots, \mu_{n}\right)$ of $L(\bar{s}, y)$ such that $\left(s, \lambda_{1}, \cdots, \lambda_{n}\right) \rightarrow\left(\bar{s}, \mu_{1}, \cdots, \mu_{n}\right)$ is a generic specialization over $\mathcal{F}$ and the field of constants of $\mathcal{F}\left\langle s, \bar{s}, \lambda_{1}, \cdots, \lambda_{n}, \mu_{1}, \cdots, \mu_{n}\right\rangle$ is $C$.

Proof. Let $\left(\omega_{1}, \cdots, \omega_{n}\right)$ be a fundamental system of zeros of $L(\bar{s}, y)$ such that the field of constants of $\mathcal{F}\left\langle s, \bar{s}, \lambda_{1}, \cdots, \lambda_{n}, \omega_{1}, \cdots, \omega_{n}\right\rangle$ is $C$. Let $\left(s, \lambda_{1}, \cdots, \lambda_{n}\right) \rightarrow\left(\bar{s}, \pi_{1}, \cdots, \pi_{n}\right)$ be a generic specialization over $\mathcal{F}$ (extending the generic specialization $s \rightarrow \bar{s}$ over $\mathcal{F})$. Then $\mathcal{F}\left\langle\bar{s}, \omega_{1}, \cdots, \omega_{n}\right\rangle, \mathcal{F}\left\langle\bar{s}, \pi_{1}, \cdots, \pi_{n}\right\rangle$ are P.V.E. of $\mathcal{F}\langle\bar{s}\rangle$ with $\pi_{i}=\sum_{j=1}^{n} b_{i j} \omega_{j}$ where $b_{i j} \in D \supseteq C$. By Corollary 2 there exists a generic specialization $\left(\pi_{1}, \cdots, \pi_{n}\right) \rightarrow\left(\mu_{1}, \cdots, \mu_{n}\right)$ over $\mathcal{F}\langle\bar{s}\rangle$ where . $\mu_{i}=\sum_{j=1}^{n} a_{i j} \omega_{j}$ with $a_{i j} \in C$; so that the field of constants of

$$
\mathcal{F}\left\langle s, \bar{s}, \lambda_{1}, \cdots, \lambda_{n}, \mu_{1}, \cdots, \mu_{n}\right\rangle
$$

is $C$. Also, $\left(s, \lambda_{1}, \cdots, \lambda_{n}\right) \rightarrow\left(\bar{s}, \pi_{1}, \cdots, \pi_{n}\right) \rightarrow\left(\bar{s}, \mu_{1}, \cdots, \mu_{n}\right)$ are both generic specializations over $\mathcal{F}$. Hence $\left(s, \lambda_{1}, \cdots, \lambda_{n}\right) \rightarrow\left(\bar{s}, \mu_{1}, \cdots, \mu_{n}\right)$ is a generic specialization over $\mathfrak{F}$.

2. Analytic specializations. A specialization $\left(t_{1}, \cdots, t_{r}\right) \rightarrow\left(\bar{t}_{1}, \cdots, \bar{t}_{r}\right)$ over $\mathcal{F}$ will be called analytic if there exist $r$ formal power series $\mu_{j}=\overline{\bar{t}}_{j}+\sum_{i=1}^{\infty} f_{i j} \beta^{i}$ $(j=1, \cdots, r)$, with coefficients $f_{i j}$ in some differential field extension $\mathcal{G}$ of $\mathcal{F}$, in a constant $\beta$ transcendental over $\mathcal{G}$, such that $\left(t_{1}, \cdots, t_{r}\right) \rightarrow\left(\mu_{1}, \cdots, \mu_{r}\right)$ is a generic specialization over $\mathcal{F}$. If $t_{1}, \cdots, t_{r}$ are differentially algebraically independent over $\mathcal{F}$ any specialization $\left(t_{1}, \cdots, t_{r}\right) \rightarrow\left(\bar{t}_{1}, \cdots, \bar{t}_{r}\right)$ is analytic, since $\left(t_{1}, \cdots, t_{r}\right) \rightarrow\left(\bar{t}_{1}+z_{1} \beta, \cdots, \bar{t}_{r}+z_{r} \beta\right)$, where $z_{1}, \cdots, z_{r}$ are $r$ new differential indeterminates, is a generic specialization over $\mathcal{F}$.

LemMa 2. Let $F(y) \in \mathcal{F}\{y\}$ be an irreducible differential polynomial of order $n$. Let $t$ be a generic zero of the general component of $F(y)$. Let $t \rightarrow \bar{t}$ be any specialization over $\mathcal{F}$ such that the differential polynomial $K(z)$ formed by the sum of terms of lowest degree of $F(\bar{t}+z) \in \mathcal{F}\langle\bar{t}\rangle\{z\}$ is of order $n$. Then the specialization $t \rightarrow \bar{t}$ is an analytic specialization over $\mathcal{F}$.

Proof. Let $M(z)$ be an irreducible factor of $K(z)$ of order $n$ and let $f_{1}$ be a generic zero of the general component of $M(z)$; then by the Ritt power series process (Ritt [3]) there exists a zero $u$ of $F(\bar{t}+z)$ of the form $u=f_{1} \beta$ $+\sum_{i=2}^{\infty} f_{i} \beta^{\mu_{i}}$ where the $\mu_{i}$ are fractions with a common denominator such that $1<\cdots<\mu_{i}<\mu_{i+1}$. Now, if any differential polynomial $P(z) \in \mathcal{F}\langle\bar{t}\rangle\{z\}$ vanishes for $z=u$, the sum of the terms of lowest degree must vanish for $z=f_{1}$; since $f_{1}$ can not satisfy any differential equation of order less than $n$. neither can $u$. Also, $\bar{t}+u=\bar{t}+\sum_{i=1}^{\infty} f_{i} \beta^{\mu_{i}}$ is a zero of $F(y)$. Suppose there existed a differential polynomial $P(y) \in \mathcal{F}\{y\}$ of order less than $n$ which vanished for $y=\bar{t}+u$; then $P(\bar{t}+z) \in \mathcal{F}\langle\bar{t}\rangle\{\dot{z}\}$. would be of order less than $n$ and 
would vanish for $z=u$, which is impossible. Hence $\bar{t}+u$ is a generic zero of the general component of $F(y)$. Since the $\mu_{i}$ have a common denominator we can replace $\beta$ by a power of itself to obtain a power series $\bar{t}+\cdots$ with the required properties.

Corollary 1. Let $t_{1}, \cdots, t_{r}, \bar{t}_{1}, \cdots, \bar{t}_{r}$ be elements of some differential field extension of $\mathcal{F}$ and let $\left(t_{1}, \cdots, t_{r-1}\right) \rightarrow\left(\bar{t}_{1}, \cdots, \bar{t}_{r-1}\right)$ be an analytic specialization over $\mathcal{F}$. Let $t_{r}$ be a generic zero of the general component of an irreducible differential polynomial

$$
F\left(t_{1}, \cdots, t_{r-1}, y\right) \in \mathcal{F}\left\{t_{1}, \cdots, t_{r-1}, y\right\}
$$

over $\mathcal{F}\left\langle t_{1}, \cdots, t_{r-1}\right\rangle$. Let $F$ be of order $n$ in $y$. Let $\left(t_{1}, \cdots, t_{r}\right) \rightarrow\left(\bar{t}_{1}, \cdots, \bar{t}_{r}\right)$ be a specialization over $\mathcal{F}$ such that the differential polynomial $K(z)$ formed by the sum of terms of lowest degree in $F\left(\bar{t}_{1}, \cdots, \bar{t}_{r-1}, \bar{t}_{r}+z\right)$ is of order $n$. Then the specialization $\left(t_{1}, \cdots, t_{r}\right) \rightarrow\left(\bar{t}_{1}, \cdots, \bar{t}_{r}\right)$ over $\mathcal{F}$ is analytic.

Proof. Let $\left(t_{1}, \cdots, t_{r-1}\right) \rightarrow\left(u_{1}, \cdots, u_{r-1}\right), u_{j}=\bar{t}_{j}+\sum_{i=1}^{\infty} f_{i j} \beta^{j}(j=1, \cdots$, $r-1)$, be a generic specialization over $\mathcal{F}$. Let $v \beta^{s}$ be the term of lowest degree in $\beta$ in $F\left(u_{1}, \cdots, u_{r-1}, \bar{t}_{r}\right)$. Let $M(z)$ be an irreducible factor of order $n$ of $K(z)+v \in \mathcal{F}\left\langle t_{1}, \cdots, t_{r},\left(f_{i j}\right)\right\rangle\{z\}$. Let $f_{1 r}$ be a generic zero of the general component of $M(z)$ and let $\mu_{1}=s m^{-1}$, or 1 according as $s \neq 0$ or $s=0$ where $m$ is the degree of $K(z)$. By the Ritt power series process there exists a zero $u_{r}$ of $F\left(u_{1}, \cdots, u_{r-1}, y\right)$ of the form $u_{r}=\bar{t}_{r}+f_{1 r} \beta^{\mu_{1}}+\sum_{i=2}^{\infty} f_{i r} \beta^{\mu_{i}}$ where the $\mu_{i}$ are fractions with a common denominator such that $\mu_{i}<\mu_{i+1}$. By the same argument as above the specialization $\left(t_{1}, \cdots, t_{r}\right) \rightarrow\left(u_{1}, \cdots, u_{r}\right)$ over $\mathcal{F}$ is generic so that the specialization $\left(t_{1}, \cdots, t_{r}\right) \rightarrow\left(\bar{t}_{1}, \cdots, \bar{t}_{r}\right)$ is analytic.

CoRollary 2. Let $t_{1}, \cdots, t_{r}, \bar{t}_{1}, \cdots, \bar{t}_{r-1}$ be as in Corollary 1 , and let $\bar{t}_{r}$ be a nonsingular solution of $F\left(\bar{t}_{1}, \cdots, \bar{t}_{r-1}, y\right) \in \mathcal{F}\left\langle\bar{t}_{1}, \cdots, \bar{t}_{r-1}\right\rangle\{y\}$. Then the specialization $\left(t_{1}, \cdots, t_{r}\right) \rightarrow\left(\bar{t}_{1}, \cdots, \bar{t}_{r}\right)$ over $\mathcal{F}$ is analytic.

Proof. Let $S(y) \in \mathscr{F}\left\langle\bar{t}_{1}, \cdots, \bar{t}_{r-1}\right\rangle\{y\}$ be the separant of $F\left(\bar{t}_{1}, \cdots, \bar{t}_{r-1}, y\right)$. Then $F\left(\bar{t}_{1}, \cdots, \bar{t}_{r-1}, \bar{t}_{r}+z\right)=S\left(\bar{t}_{r}\right) z^{(n)}+\cdots$. Since $S(\bar{t}) \neq 0$ the sum of terms of lowest degree in $F\left(\bar{t}_{1}, \cdots, \bar{t}_{r-1}, \bar{t}_{r}+z\right)$ is of order $n$. By Corollary 1 the specialization $\left(t_{1}, \cdots, t_{r}\right) \rightarrow\left(\bar{t}_{1}, \cdots, t_{r}\right)$ over $\mathcal{F}$ is analytic.

Example 1. Let $\mathcal{F}=C$, let $F(y)=y^{\prime 2}-4 y^{3}$ and let $t$ be a generic zero of $\{F\}(\{F\}$ is a prime differential ideal, for 0 is the only singular zero of $F$ and by the low power theorem (Ritt [3]) 0 is in the general manifold of $F$ ), and let $\bar{t}=0$ then $t \rightarrow \bar{t}$ is an analytic specialization over $\mathcal{F}$. For $u=0+\beta^{2}(1-\beta x)^{-2}$ $=\sum_{n=0}^{\infty}(n+1) x^{n} \beta^{n+2}$ (where $\left.x^{\prime}=1\right)$ is a generic zero of $\{F\}$.

The following example shows that the conditions imposed in Lemma 2 on $\bar{t}$ for $t \rightarrow \bar{t}$ to be an analytic specialization over $\mathcal{F}$ are not superfluous.

EXAmple 2. Let $F=C$ and let $F(y)=y y^{\prime \prime}+y^{\prime} .\{F\}$ is a prime ideal for the same reason as given in Example 1. Hence 0 is in the general manifold of $F$. Let $u=0+\sum f_{i} \beta^{i}$ be a zero of $F(y)$; then $\left(f_{i}\right)_{1 \leq i<\infty}$ are constants. Indeed, $f_{1}$ must be a zero of $y^{\prime}$ which is in the term of lowest degree in $F(y)$, so that $f_{1}$ 
must be a constant; assuming $f_{i}(i=1, \cdots, n-1)$ are constants, then $F(u)$ $=\left(\sum_{r=1}^{\infty} f_{i} \beta^{i}\right)\left(\sum_{i=n}^{\infty} f_{i}^{\prime \prime} \beta^{i}\right)+\sum_{i=n}^{\infty} f_{i}^{\prime} \beta^{i}$, the coefficient of $\beta^{n}$ is $f_{i}^{\prime}$, so that $f_{i}$ is a constant. Hence $u$ is a constant and can not be a generic zero of $\{F\}$. Note, however, that by Corollary 2 to Lemma 2 if $c$ is any nonzero constant there exists a generic zero $u$ of $\{F\}$ of the form $u=c+\sum_{i=1}^{\infty} f_{i} \beta^{i}$.

\section{Specialization of homogeneous linear differential equations.}

THEOREM 1. Let $L(t, y)=a_{0}(t) y^{(n)}+\cdots+a_{n}(t) y \in \mathcal{F}\{t, y\}$. Let $t \rightarrow \bar{t}$ be an analytic specialization over $\mathcal{F}$ such that $a_{0}(\bar{t}) \neq 0$ and the field of constants of $\mathcal{F}\langle\bar{t}\rangle$ is $C$. Then for any fundamental system of zeros $\left(\omega_{1}, \cdots, \omega_{n}\right)$ of $L(\bar{t}, y)$ there exists a fundamental system of zeros $\left(\pi_{1}, \cdots, \pi_{n}\right)$ of $L(t, y)$ such that $\left(t, \pi_{1}, \cdots, \pi_{n}\right) \rightarrow\left(\bar{t}, \omega_{1}, \cdots, \omega_{n}\right)$ is an analytic specialization over $\mathcal{F}$.

Proof. Let $t \rightarrow \bar{t}+\sum_{i=1}^{\infty} f_{i} \beta^{i}$ be a generic specialization over $\mathcal{F}$ and let

$$
L\left(\bar{t}+\sum_{i=1}^{\infty} f_{i} \beta^{i}, y\right)=\sum_{i=0}^{n} \sum_{j=0}^{\infty} g_{i j} \beta^{i} y^{(n-i)}
$$

where each $g_{i j} \in \mathcal{F}\left\langle\bar{t}, \quad\left(f_{i}\right)_{1 \leq i<\infty}\right\rangle$. Let $\lambda_{k}=\omega_{k}+\sum_{m=1}^{\infty} h_{k m} \beta^{m} \quad\left(h_{k m}\right.$ to be determined). Then

$$
\begin{aligned}
L(\bar{t} & \left.+\sum_{i=1}^{\infty} f_{i} \beta^{i}, \lambda_{k}\right)=\sum_{i=0}^{n} \sum_{j=1}^{\infty} g_{i j} \beta^{i}\left(\omega_{k}^{(n-i)}+\sum_{n=1}^{\infty} h_{k m} \beta^{m}\right) \\
& =L\left(\bar{t}, \omega_{k}\right)+\sum_{i=0}^{n} \sum_{j=0}^{\infty} g_{i j} \beta^{i} \omega_{k}^{(n-i)}+\sum_{i=0}^{n} \sum_{j=0}^{\infty} g_{i j} \sum_{m=1}^{\infty} h_{k m}^{(n-i)} \beta^{j+m} \\
& =\sum_{i=0}^{n} \sum_{s=1}^{\infty}\left(g_{i s} \omega_{k}^{(n-i)}+\sum_{j+m=s} g_{i j} h_{k m}^{(n-i)}\right) \beta^{s} \\
& =\sum_{s=1}^{\infty}\left[\sum_{i=0}^{n}\left(\sum_{j+m=s} g_{i j} h_{k m}^{(n-i)}+g_{i s} \omega_{k}^{(n-i)}\right)\right] \beta^{s} \\
& =\sum_{s=1}^{\infty}\left[\sum_{i=0}^{n} g_{i 0} h_{k s}^{(n-i)}+\sum_{i=0}^{n}\left(\sum_{i+m=s ; m<s} g_{i j} h_{k m}^{(n-i)}+g_{i s} \omega_{k}^{(n-i)}\right)\right] \beta^{s} \\
& =\sum_{s=1}^{\infty}\left[L\left(\bar{t}, h_{k s}\right)+\sum_{i=0}^{n} \sum_{j+m=s ; m<s} g_{i j} h_{k m}^{(n-i)}+g_{i s} \omega_{k}^{(n-i)}\right] \beta^{s} .
\end{aligned}
$$

We choose $h_{k s}$ successively $(s=1,2, \cdots)$ to be solutions of

$$
L(\bar{t}, y)=-\sum_{i=0}^{n}\left(\sum_{j+m=s ; m<s} g_{i j} h_{k m}^{(n-i)}+g_{i s} \omega_{k}^{(n-i)}\right) \quad(k=1, \cdots, n) .
$$

Then $L\left(\bar{t}+\sum f_{i} \beta^{i}, \lambda_{k}\right)=0(k=1, \cdots, n)$ and the Wronskian $W\left(\lambda_{1}, \cdots, \lambda_{n}\right)$ $\neq 0$, for $W\left(\omega_{1}, \cdots, \omega_{n}\right) \neq 0$. Now any differential polynomial

$$
P\left(\bar{t}+\sum f_{i} \beta^{i}, y_{1}, \cdots, y_{n}\right) \in \mathcal{F}\left\{\bar{t}+\sum f_{i} \beta^{i}, y_{1}, \cdots, y_{n}\right\}
$$


which vanishes for $y_{i}=\lambda_{i}(i=1, \cdots, n)$ must have the property that $P\left(\bar{t}, \omega_{1}, \cdots, \omega_{n}\right)=0$. Since $t \rightarrow \bar{t}+\sum_{i=1}^{\infty} f_{i} \beta^{i}$ is a generic specialization over $\mathcal{F}$ there exists $\left(\pi_{1}, \cdots, \pi_{n}\right)$ such that $\left(t, \pi_{1}, \cdots, \pi_{n}\right) \rightarrow\left(\bar{t}+\sum f_{i} \beta^{i}, \lambda_{1}, \cdots, \lambda_{n}\right)$ is a generic specialization over $\mathcal{F}$. Hence $\left(t, \pi_{1}, \cdots, \pi_{n}\right) \rightarrow\left(\bar{t}, \omega_{1}, \cdots, \omega_{n}\right)$ is an analytic specialization over $\mathcal{F}$.

Note. The $h_{k s}$ are solutions of linear differential equations over

$$
\mathfrak{F}\left\langle\bar{t},\left(f_{i}\right)_{1 \leq i<\infty}, h_{k 1}, \cdots, h_{k, s-1}\right\rangle .
$$

Hence it is possible to choose the $h_{k s}$ such that the field of constants of $\mathcal{F}\left\langle\bar{t},\left(f_{i}\right)_{1 \leq i<\infty}, h_{k s ; 1 \leq s<\infty, 1 \leq k \leq n}\right\rangle$ is contained in $B$ where $B$ is the algebraic closure of $\mathcal{F}\left\langle\bar{t},\left(f_{i}\right)_{1 \leq i<\infty}\right\rangle$.

If $\mathcal{G}$ is a differential field with an algebraically closed field of constants, and $\left(\pi_{1}, \cdots, \pi_{n}\right)$ is a fundamental system of zeros of $L(y)=a_{0} y^{(n)}+\cdots$ $+a_{n} y \in \mathcal{G}\{y\}$ such that $\mathcal{G}\left\langle\pi_{1}, \cdots, \pi_{n}\right\rangle$ is a P.V.E. of $\mathcal{G}$; then by the algebraic matric group of $\mathcal{G}\left\langle\pi_{1}, \cdots, \pi_{n}\right\rangle$ over $\mathcal{G}$ we shall always mean (without stating it explicitly) the algebraic matric group associated with the fundamental system of zeros $\left(\pi_{1}, \cdots, \pi_{n}\right)$.

THEOREM 2. Let $L(t, y)=a_{0}(t) y^{(n)}+\cdots+a_{n}(t) y \in \mathcal{F}\{t, y\}$, let $t \rightarrow \tilde{t}$ $=\bar{t}+\sum_{i=1}^{\infty} f_{i} \beta^{i}$ be a generic specialization over $\mathcal{F}$ such that $a_{0}(\bar{t}) \neq 0$, let $\left(\omega_{1}, \cdots, \omega_{n}\right)$ be a fundamental system of zeros of $L(\bar{t}, y)$ such that the field of constants of $\mathcal{F}\left\langle\bar{t},\left(f_{i}\right)_{1 \leqq i<\infty}, \omega_{1}, \cdots, \omega_{n}\right\rangle$ is $C$, and let $H^{C}$ be the algebraic matric group of $\mathcal{F}\left\langle\bar{t},\left(f_{i}\right), \omega_{1}, \cdots, \omega_{n}\right\rangle$ over $\mathcal{F}\left\langle\bar{t},\left(f_{i}\right)\right\rangle$. Then there exists a fundamental system of zeros $\left(\pi_{1}, \cdots, \pi_{n}\right)$ of $L(t, y)$ and an algebraically closed field of constants $E \supset C$ such that:

(1) The field of constants of $\mathcal{F}\langle t, E\rangle$ is $E$, and $\mathcal{F}\left\langle t, E, \pi_{1}, \cdots, \pi_{n}\right\rangle$ is a P.V.E. of $\mathcal{F}\langle t, E\rangle$, with the algebraic matric group denoted by $G^{E}$.

(2) $\left(t, \pi_{1}, \cdots, \pi_{n}\right) \rightarrow\left(\bar{t}, \omega_{1}, \cdots, \omega_{n}\right)$ is an analytic specialization over $\mathcal{F}$.

(3) There exists a subgroup $K^{E}$ of $G^{E}$ such that the specialization in (2) induces simultaneously a specialization $\left(b_{i j}\right) \rightarrow\left(\bar{b}_{i j}\right)$ over $\mathcal{F}$ of all the elements $\left(b_{i j}\right)$ of $K^{E}$ such that the mapping $\left(b_{i j}\right) \rightarrow\left(\bar{b}_{i j}\right)$ is a group homomorphism of $K^{E}$ onto $H^{C}$.

Proof. By Theorem 1 there exists a fundamental system of zeros $\left(\pi_{1}, \cdots, \pi_{n}\right)$ of $L(t, y)$ such that $\left(t, \pi_{1}, \cdots, \pi_{n}\right) \rightarrow\left(\bar{t}, \omega_{1}, \cdots, \omega_{n}\right)$ is an analytic specialization over $\mathcal{F}$; therefore there exists a generic specialization $\left(t, \pi_{1}, \cdots, \pi_{n}\right) \rightarrow\left(\bar{t}+\sum_{i=1}^{\infty} f_{i} \beta^{i}, \lambda_{1}, \cdots, \lambda_{n}\right)$ over $\mathcal{F}$, where $\lambda_{j}=\omega_{j}+\sum_{i=1}^{\infty} g_{i j} \beta^{i}$ $(j=1, \cdots, n)$, where the field of constants of $\mathcal{F}\left\langle\bar{t},\left(f_{i}\right)_{1 \leq i<\infty},\left(g_{i j}\right)_{1 \leq i<\infty, 1 \leq j<\infty}\right\rangle$ is $C$.

Let the field of constants of $\mathcal{F}\left\langle\tilde{t}, \lambda_{1}, \cdots, \lambda_{n}\right\rangle$ be $B$. If $b \in B$ then

$$
\begin{gathered}
b \in \mathcal{F}\left\langle\bar{t},\left(f_{i}\right)_{1 \leq i<\infty},\left(g_{i j}\right)_{1 \leq i<\infty, 1 \leq i \leq n}\right\rangle((\beta)) ; \\
\left(b=\sum r_{k} \beta^{k}, b^{\prime}=\sum r_{k}^{\prime} \beta^{k}=0, r_{k}^{\prime}=0, r_{k} \in C\right)
\end{gathered}
$$

so that 


$$
b \in C((\beta)) .
$$

Let $E$ be the algebraic closure of $C((\beta))$; then the elements of $E$ are fractional power series in $\beta$, with coefficients in $C$, having the property that only a finite number of terms with negative exponents have nonzero coefficients, and that the set of all exponents which appear in terms with nonzero coefficients have a common denominator. Now $\mathscr{F}\left\langle E, t, \lambda_{1}, \cdots, \lambda_{n}\right\rangle$ is a P.V.E. of $\mathcal{F}\langle E, t\rangle$ (Kolchin [2]). Let $G^{E}$ denote the algebraic matric group of automorphisms of this extension.

Let $\left(a_{j k}\right) \in H^{c}$. Then $\left(\omega_{k}\right) \rightarrow\left(\sum_{j=1}^{n} a_{j k} \omega_{j}\right)(k=1, \cdots, n)$ is a generic specialization over $\mathcal{F}\left\langle\bar{t},\left(f_{i}\right)_{1 \leq i<\infty}\right\rangle$. This can be extended to a generic specialization

$$
\left(\left(\omega_{k}\right)_{1 \leq k \leq n},\left(g_{i j}\right)_{1 \leq i<\infty, 1 \leq j \leq n}\right) \rightarrow\left(\left(\sum_{j=1}^{n} a_{j k} \omega_{j}\right)_{1 \leq k \leq n},\left(s_{i j}\right)_{1 \leq i<\infty, 1 \leq i \leq n}\right)
$$

over $\mathcal{F}\left\langle\bar{t},\left(f_{i}\right)_{1 \leq i<\infty}\right\rangle$. Obviously, then

$$
\left(\omega_{k}+\sum_{i=1}^{\infty} g_{i k} \beta^{i}\right)_{1 \leq k \leq n} \rightarrow\left(\sum_{j=1}^{n} a_{j k} \omega_{j}+\sum_{i=1}^{\infty} s_{i k} \beta^{i}\right)_{1 \leq k \leq n}
$$

is a generic specialization over $\mathscr{F}\left\langle\bar{t}+\sum_{i=1}^{\infty} f_{i} \beta^{i}\right\rangle=\mathscr{F}\langle\bar{t}\rangle$. Since each $g_{i j}, s_{i j}$ $(1 \leqq i<\infty, 1 \leqq j \leqq n)$ is a zero of a linear differential polynomial we may assume, by Corollary 3 of Lemma 1, that the field of constants of $\mathcal{F}\left\langle\omega_{1}, \cdots, \omega_{n},\left(g_{i j}\right)_{1 \leq i<\infty, 1 \leq j \leq n} ;\left(s_{i j}\right)_{1 \leq i<\infty, 1 \leq j \leq n}\right\rangle$ is $C$.

Let $\sigma$ be the isomorphism of $\mathcal{F}\left\langle t, \lambda_{1}, \cdots, \lambda_{n}\right\rangle$ over $\mathcal{F}\langle t\rangle$ such that

$$
\sigma \lambda_{k}=\sum_{j=1}^{n} a_{j k} \omega_{j}+\sum_{i=1}^{\infty} s_{i k} \beta^{i} \quad(1 \leqq k \leqq n) .
$$

Since $\lambda_{1}, \cdots, \lambda_{n}$ is a fundamental system of zeros of $L(t, y)$ there exist constants $b_{i j}$ such that

$$
\sigma \lambda_{k}=\sum_{j=1}^{n} b_{j k} \lambda_{j}=\sum_{j=1}^{n} b_{j k}\left(\omega_{j}+\sum_{i=1}^{\infty} g_{i j} \beta^{i}\right) .
$$

Differentiating we find $\sum_{j=1}^{n} b_{j k} \lambda_{j}^{(m)}=\sigma \lambda_{k}^{(m)}(0 \leqq m \leqq n-1)$. Solving these linear equations we obtain

$$
\begin{aligned}
b_{j k} & =\frac{W\left(\lambda_{1}, \cdots, \lambda_{j-1}, \sigma \lambda_{k}, \lambda_{j+1}, \cdots, \lambda_{n}\right)}{W\left(\lambda_{1}, \cdots, \lambda_{n}\right)} \\
& =\frac{W\left(\omega_{1}, \cdots, \omega_{j-1}, \sum_{m=1}^{n} a_{m k} \omega_{m}, \omega_{j+1}, \cdots, \omega_{n}\right)+\cdots}{W\left(\omega_{1}, \cdots, \omega_{n}\right)+\cdots} \\
& =a_{j k}+\cdots,
\end{aligned}
$$

where the unwritten terms all have degree $>0$ in $\beta$. Thus 


$$
b_{j k} \in \mathcal{F}\left\langle\omega_{1}, \cdots, \omega_{n},\left(g_{i j}\right),\left(s_{i j}\right)\right\rangle((\beta)),
$$

whence (since $b_{j k}$ is a constant), $b_{j k} \in C((\beta))$. 'Moreover, every term of $b_{j k}$ of degree $<0$ in $\beta$ has coefficient 0 , and the coefficient of degree zero is $a_{j k}$ :

$$
b_{j k}=a_{j k}+\sum_{i=1}^{\infty} c_{i j k} \beta^{i} \quad\left(c_{i j k} \in C\right) .
$$

Therefore $\sigma=\left(b_{j k}\right)$ is an element of the algebraic matric group of $\mathcal{F}\langle E, t$, $\left.\lambda_{1}, \cdots, \lambda_{n}\right\rangle$ over $\mathcal{F}\langle E, t\rangle$, that is $\sigma \in G^{E}$.

Let $K^{E}$ be the set of all elements $\left(b_{j k}\right) \in G^{E}$ such that each $b_{j k}$ is of the form $\bar{b} j_{k}+\sum_{i=1}^{\infty} c_{i j k} \beta^{i}$, where $c_{i j k} \in C$ and $\left(\bar{b}_{j k}\right) \in H^{c}$; then $K^{E}$ is a group and the mapping $\left(b_{j k}\right) \rightarrow\left(\bar{b}_{j k}\right)$ is a group homomorphism of $K^{E}$ onto $H^{c}$.

Since $\left(t, \pi_{1}, \cdots, \pi_{n}\right) \rightarrow\left(t, \lambda_{1}, \cdots, \lambda_{n}\right)$ is a generic specialization over $\mathcal{F}$ we may identify the field of constants of $\mathcal{F}\left\langle t, \pi_{1}, \cdots, \pi_{n}\right\rangle$ with the field of constants of $\mathcal{F}\left\langle t, \lambda_{1}, \cdots, \lambda_{n}\right\rangle$, so that the group of $\mathcal{F}\left\langle t, E, \pi_{1}, \cdots, \pi_{n}\right\rangle$ over $\mathscr{F}\langle t, E\rangle$ is $G^{E}$.

EXAMPLE 1. Let $\mathcal{F}=C=$ field of complex numbers and let $t$ be a transcendental constant over $\mathcal{F}$. Let $\bar{t}=0$ then $t \rightarrow 0+\beta$ is a generic specialization over F. Let $L(t, y)=y^{\prime \prime}-3 t y^{\prime}+2 t^{2} y, L(0+\beta, y)=y^{\prime \prime}-3 \beta y^{\prime}+2 \beta^{2} y$ and $L(\bar{t}, y)=y^{\prime \prime}$. Let $\omega_{1}=1, \omega_{2}=x, \pi_{1}=e^{\beta x}, \pi_{2}=\left(e^{2 \beta x}-e^{\beta x}\right) \beta^{-1}$ then

$$
\pi_{1}=\omega_{1}+\sum_{i=1}^{\infty} \frac{x^{i} \beta^{i}}{i !}, \quad \pi_{2}=\omega_{2}+\sum_{i=1}^{\infty} \frac{(2 x)^{i+1}-x^{i+1}}{(i+1) !} \beta^{i} .
$$

Let $E$ be the algebraic closure of $C((\beta))$; then the algebraic matric group of $E\left\langle e^{\beta x}, e^{2 \beta x}\right\rangle$ over $E$ consists of the set of all matrices

$$
\left(\begin{array}{ll}
a & 0 \\
0 & a^{2}
\end{array}\right)
$$

with $a \in E$

and $a \neq 0$. Hence the algebraic matric group $G^{E}$ of $E\left\langle\pi_{1}, \pi_{2}\right\rangle$ over $E$ consists of the set of all matrices

$$
\left(\begin{array}{cc}
a & \left(a^{2}-a\right) \beta^{-1} \\
0 & a^{2}
\end{array}\right) \quad \text { with } a \in E \text { and } a \neq 0
$$

which is the same as the set of all matrices

$$
\left(\begin{array}{cc}
1+b \beta & b+b^{2} \beta \\
0 & (1+b \beta)^{2}
\end{array}\right) \quad \text { with } b \in E \text { and } b \neq-\beta^{-1} \text {. }
$$

The algebraic matric group $H^{C}$ of $\mathcal{F}\langle 1, x\rangle$ over $\mathcal{F}$ consists of the set of all matrices

$$
\left(\begin{array}{ll}
1 & c \\
0 & 1
\end{array}\right) \quad c \in C
$$


Here $K^{E}$ consists of those matrices

$$
\left(\begin{array}{cc}
1+b \beta & b+b^{2} \beta \\
0 & (1+b \beta)^{2}
\end{array}\right)
$$

for which $b$ has order $\geqq 0$ in $\beta$.

The algebraic matric group $H^{c}$ of Theorem 2 is the group of all automorphisms of $\mathcal{F}\left\langle\bar{t},\left(f_{i}\right)_{1 \leq i<\infty}, \omega_{1}, \cdots, \omega_{n}\right\rangle$ over $\mathcal{F}\left\langle\bar{t},\left(f_{i}\right)\right\rangle . H^{c}$ is a subgroup of the algebraic matric group $N^{C}$ of automorphisms of $\mathcal{F}\left\langle\bar{t}, \omega_{1}, \cdots, \omega_{n}\right\rangle$ over $\mathcal{F}\langle\bar{t}\rangle$. The following example will show that if $\left(\bar{b}_{i j}\right) \in N^{c}$ and $\left(b_{i j}\right) \notin H^{c}$ there may not exist $\left(b_{i j}\right) \in G^{E}$ such that $\left(t, \pi_{1}, \cdots, \pi_{n},\left(b_{i j}\right)\right) \rightarrow\left(\bar{t}, \omega_{1}, \cdots, \omega_{n},\left(b_{i j}\right)\right)$ is a specialization over $\mathcal{F}$.

EXAMPLE 2. Let $\mathcal{F}=C=$ field of complex numbers. Let $t=e^{x}, \bar{t}=0, \tilde{t}=0+f \beta$ $=0+e^{x} \beta$ and let $L(t, y)=y^{\prime \prime}-\left[\left(1+2^{1 / 2}\right) e^{x}+1\right] y^{\prime}+2^{1 / 2} e^{2 x} y$; then $t \rightarrow \bar{t}$ is an analytic specialization over $\mathcal{F}$. For the differential polynomial, over $\mathcal{F}$, of lowest order which vanishes for $y=t$ is $y^{\prime}-y$ so that $t \rightarrow \tilde{t}$ is a generic specialization over $\mathcal{F}$. $L(t, y)$ has a fundamental system of zeros $\left(e^{e^{x}}, e^{(2)^{1 / 2} e^{x}}\right)$. The algebraic matric group of $\mathcal{F}\left\langle e^{x}, e^{e^{x}}, e^{2^{1 / 2} e^{x}}\right\rangle$ over $\mathcal{F}\left\langle e^{x}\right\rangle$ is the full diagonal group; for the differential equation of lowest order that $e^{e^{x}}$ satisfies over $\mathfrak{F}\left\langle e^{x}\right\rangle$ is $y^{\prime}-e^{x} y=0$, and the differential equation of lowest order that $e^{2^{1 / 2} e^{x}}$ satisfies over $\mathcal{F}\left\langle e^{x}, e^{e^{x}}\right\rangle$ is $y^{\prime}-2^{1 / 2} e^{x} y=0$. Similarly, the algebraic matric group of $\mathcal{F}\left\langle\tilde{t}, e^{\beta e^{x}}, e^{2^{1 / 2} \beta e^{x}}\right\rangle$ over $\mathcal{F}\langle\tilde{t}\rangle$ is the full diagonal group, since $\left(t, e^{e^{x}}, e^{2^{1 / 2} e^{x}}\right)$ $\rightarrow\left(\tilde{t}, e^{\beta e^{x}}, e^{2^{1 / 2} \beta e^{x}}\right)$ is a generic specialization over $\mathcal{F}$. Now, $L(\bar{t}, y)=y^{\prime \prime}-y^{\prime}$ which has $\omega_{1}=1 \omega_{2}=e^{x}$ as a fundamental system of zeros.

Let

$$
\begin{aligned}
& \pi_{1}=e^{\beta e^{x}}=1+\sum_{i=1}^{\infty} \frac{e^{i x} \beta^{i}}{i !} \\
& \pi_{2}=\left(e^{2^{1 / 2} \beta_{e}^{x}}-e^{\beta c x}\right)\left(2^{1 / 2}+1\right) \beta^{-1}=e^{x}+\sum_{i=2}^{\infty} \frac{\left[\left(2^{i}\right)^{1 / 2}-1\right] e^{i x} \beta^{i-1}}{\left(2^{1 / 2}-1\right) i !}
\end{aligned}
$$

so that $\left(t, \pi_{1}, \pi_{2}\right) \rightarrow\left(0, \omega_{1}, \omega_{2}\right)$ is a specialization over $\mathcal{F} . \mathcal{F}\left\langle\tilde{t}, \pi_{1}, \pi_{2}\right\rangle$ is not a P.V.E. of $\mathcal{F}\langle t\rangle$, for $\beta$ which is transcendental over $\mathcal{F}\langle t\rangle$ belongs to $F\left\langle t, \pi_{1}, \pi_{2}\right\rangle$, $\left(\beta=\pi_{1} t\left(\pi_{2}^{\prime}-2^{1 / 2} \pi_{2} t\right)^{-1}\right)$. Let $E$ be the algebraic closure of $C((\beta))$; then the algebraic matric group $G^{E}$ of $E\left\langle t, \pi_{1}, \pi_{2}\right\rangle$ over $E\langle t\rangle$ consists of the set of all matrices

$$
\left(\begin{array}{cc}
a & (b-a)\left(2^{1 / 2}+1\right) \beta^{-1} \\
0 & b
\end{array}\right) \quad \text { with } a, b \in E \text { and } a, b \neq 0
$$

which is the same as the set of all matrices

$$
\left(\begin{array}{cc}
1+a \beta & (b-a)\left(2^{1 / 2}+1\right) \\
0 & 1+b \beta
\end{array}\right) \quad \text { with } a, b \in E \text { and } a, b \neq-\beta^{-1} .
$$


The algebraic matric group $N^{C}$ of $\mathcal{F}\left\langle\omega_{1}, \omega_{2}\right\rangle$ over $\mathcal{F}$ is the set of all matrices of the form

$$
\left(\begin{array}{ll}
1 & 0 \\
0 & b
\end{array}\right)
$$

with $b \in C b \neq 0$.

Since $f=e^{x} \mathfrak{F}\left\langle f, \omega_{1}, \omega_{2}\right\rangle=\mathfrak{F}\langle f\rangle$ so that $H^{c}$ is reduced to the identity matrix. It is easy to see that if $\left(b_{i j}\right) \in N^{C}$ and is not the identity matrix there does not exist $\left(b_{i j}\right) \in G^{E}$ such that $\left(t, \pi_{1}, \pi_{2},\left(b_{i j}\right)\right) \rightarrow\left(z, \omega_{1}, \omega_{2},\left(\bar{b}_{i j}\right)\right)$ is a specialization over $\mathcal{F}$.

Corollary. Let the field of constants of $\mathscr{F}, \mathcal{F}\langle t\rangle$ and $\mathscr{F}\left\langle\bar{t},\left(f_{i}\right)_{1 \leq i<\infty}\right\rangle$ be $C$, let $L(t, y)$ be as in Theorem 2 , and let $\left(t, \pi_{1}, \cdots, \pi_{n}\right) \rightarrow\left(\bar{t}, \omega_{1}, \cdots, \omega_{n}\right)$ be an analytic specialization over $\mathcal{F}$, where $\mathcal{F}\left\langle t, \pi_{1}, \cdots, \pi_{n}\right\rangle$ is a P.V.E. of $\mathcal{F}\langle t\rangle$ with algebraic matric group $G$ and $\mathfrak{F}\left\langle\bar{t},\left(f_{i}\right)_{1 \leqq i<\infty}, \omega_{1}, \cdots, \omega_{n}\right\rangle$ is a P.V.E. of $\mathfrak{F}\left\langle\bar{t},\left(f_{i}\right)_{1 \leq i<\infty}\right\rangle$ with algebraic matric group $H$. Then $H \subseteq G$.

Proof. The algebraic matric group of $\mathfrak{F}\left\langle t, \pi_{1}, \cdots, \pi_{n}, E\right\rangle$ over $\mathcal{F}\langle t, E\rangle$ is the algebraic group $G^{E}$, that is, is defined by the same set II of polynomials with coefficients in $C$ as defines $G$. Let $\left(\bar{b}_{i j}\right) \in H$; by Theorem 2 there exists a $\left(b_{i j}\right) \in G^{E}$ such that $\left(b_{i j}\right) \rightarrow\left(\bar{b}_{i j}\right)$ is a specialization over $\mathcal{F}$ and hence over $C$. Since $\left(b_{i j}\right)$ is a zero of $\Pi$, so is $\left(\bar{b}_{i j}\right)$, so that $\bar{b}_{i j} \in G$.

REMARK 1. If the $\left(f_{i}\right)_{1 \leq i<\infty} \in \mathcal{F}\langle\bar{t}\rangle$ then $\mathscr{F}\left\langle\bar{t},\left(f_{i}\right)_{11_{i<\infty}}\right\rangle=\mathscr{F}\langle\bar{t}\rangle$ so that the group of $\mathcal{F}\left\langle\bar{t}, \omega_{1}, \cdots, \omega_{n}\right\rangle$ over $\mathscr{F}\langle\bar{t}\rangle$ is $H \subseteq G$. This condition is, obviously, satisfied if $\left(t_{1}, \cdots, t_{r}\right)=t$ are $r$ differential indeterminates over $\mathcal{F}$.

REMARK 2. Let the field of constants of $\mathcal{F}\langle t, \bar{t}\rangle$ be $C$ where $t \rightarrow \bar{t}$ is an analytic specialization over $\mathcal{F}$. Let $\left(\pi_{1}, \cdots, \pi_{n}\right)$ be a fundamental system of zeros of $L(t, y)=a_{0}(t) y^{(n)}+\cdots+a_{n}(t) y \in \mathcal{F}\{t, y\}$ such that $a_{0}(\bar{t}) \neq 0$ and $\mathcal{F}\left\langle t, \pi_{1}, \cdots, \pi_{n}\right\rangle$ is a P.V.E. of $\mathcal{F}\langle t\rangle$. We wish to show that except for certain singular cases the analytic specialization $t \rightarrow \bar{t}$ over $\mathcal{F}$ can be extended to an analytic specialization $\left(t, \pi_{1}, \cdots, \pi_{n}\right) \rightarrow\left(\bar{t}_{1}, \bar{\pi}_{1}, \cdots, \bar{\pi}_{n}\right)$ over $\mathcal{F}$. For, let $F_{i}\left(t, \pi_{1}, \cdots, \pi_{i-1}, y\right) \in \mathcal{F}\left\{t, \pi_{1}, \cdots, \pi_{n-i}, y\right\}$ be the irreducible differential polynomial over $\mathcal{F}\left\langle t, \pi_{1}, \cdots, \pi_{i-1}\right\rangle$ of lowest order in $y$ which vanishes for $y=\pi_{i}$. Suppose that we have already found $\left(\bar{\pi}_{1}, \cdots, \bar{\pi}_{i-1}\right)$ such that $\left(t, \pi_{1}, \cdots, \pi_{i-1}\right) \rightarrow\left(\bar{t}, \bar{\pi}_{1}, \cdots, \bar{\pi}_{i-1}\right)$ is an analytic specialization over $\mathcal{F}$ where $\left(\bar{\pi}_{1}, \cdots, \bar{\pi}_{i-1}\right)$ are linearly independent and the field of constants of $\mathfrak{F}\left\langle\bar{t}, \bar{\pi}_{1}, \cdots, \bar{\pi}_{i-1}\right\rangle$ is $C$. Let $S_{i}$ be the separant of $F_{i}$ with respect to $y$ and let $W\left(\bar{\pi}_{1}, \cdots, \bar{\pi}_{i-1}, y\right) \cdot S_{i}\left(\bar{t}, \bar{\pi}_{1}, \cdots, \bar{\pi}_{i-1}, y\right) \notin\left\{F_{i}\left(t, \bar{\pi}_{1}, \cdots, \bar{\pi}_{i-1} y\right\}\right.$. Then we may choose $\bar{\pi}_{i}$ to be a zero of $F_{i}\left(\bar{t}, \bar{\pi}_{1}, \cdots, \bar{\pi}_{i-1}, y\right)$ such that $W\left(\bar{\pi}_{1}, \cdots, \bar{\pi}_{i}\right) \cdot S_{i}\left(\bar{\pi}_{1}, \cdots, \bar{\pi}_{i}\right) \neq 0$. Furthermore $\bar{\pi}_{i}$ may be so chosen that $\mathcal{F}\left\langle\bar{t}, \bar{\pi}_{1}, \cdots, \bar{\pi}_{i}\right\rangle$ has the field of constants $C$. By Corollary 2 of Lemma 2 the specialization $\left(t, \bar{\pi}_{1}, \cdots, \pi_{i}\right) \rightarrow\left(\bar{t}, \bar{\pi}_{1}, \cdots, \bar{\pi}_{i}\right)$ over $\mathcal{F}$ is analytic.

4. Extension of specializations. Throughout the rest of this paper we shall assume that the field of constants of $\mathcal{F}, \mathcal{F}\langle t, \bar{t}\rangle$ is $C$. 
TheOREM 3. Let $L(t, y)=a_{0}(t) y^{(n)}+\cdots+a_{n}(t) y \in \mathcal{F}\{t, y\}$ and let $t \rightarrow \tilde{t}$ $=\bar{t}+\sum_{i=1}^{\infty} f_{i} \beta^{i}$ be a generic specialization over $\mathcal{F}$ such that $a_{0}(\bar{t}) \neq 0$. Let $\pi$ be any nonzero solution of $L(t, y)=0$ such that the field of constants of $\mathcal{F}\langle t, \pi\rangle$ is $C$. Then the following holds:

(1) There exists $\lambda=\beta^{r}\left(\sum_{i=0}^{\infty} g_{i} \beta^{i}\right), g_{0} \neq 0, r$ an integer, such that $(t, \pi) \rightarrow(\tilde{t}, \lambda)$ is a generic specialization over $\mathcal{F}$ :

(2) either there exists an element $\omega$ such that $(t, \pi) \rightarrow(\bar{t}, \omega)$ is a specialization over $\mathcal{F}$, where the field of constants of $\mathcal{F}\langle\bar{t}, \omega\rangle$ is $C$ or else $\left(t, \pi^{-1}\right) \rightarrow(\bar{t}, 0)$ is a specialization over $\mathcal{F}$;

(3) there exists a nonzero solution $\omega$ of $L(\bar{t}, y)=0$ such that $\left(t, \pi^{\prime} \pi^{-1}\right)$ $\rightarrow\left(\bar{t}, \omega^{\prime} \omega^{-1}\right)$ is a specialization over $\mathcal{F}$ and the field of constants of $\mathcal{F}\langle\bar{t}, \omega\rangle$ is $C$;

(4) if the field of constants of $\mathcal{F}\left\langle\bar{t},\left(f_{i}\right)_{1 \leq i<\infty}\right\rangle$ is $C$ then the specialization $(t, \pi) \rightarrow(\bar{t}, \omega)$ over $\mathcal{F}$ of $(2)$ and (3) is analytic.

Proof. Let the field of constants of $\mathcal{F}\left\langle\bar{t},\left(f_{i}\right)\right\rangle$ be $B \supseteq C$. Let $\left(\omega_{1}, \cdots, \omega_{n}\right)$ be a fundamental system of zeros of $L(\bar{t}, y)$ such that the field of constants of $\mathcal{F}\left\langle\bar{t}, \omega_{1}, \cdots, \omega_{n}\right\rangle$ is $C$. By Theorem 1 there exists a fundamental system of zeros $\lambda_{k}=\omega_{k}+\sum_{m=1}^{\infty} g_{k m} \beta^{m}(k=1, \cdots, n)$ of $L(t, y)$. We may assume that the algebraic closure of the field of constants of $\mathfrak{F}\left\langle\bar{t}, \omega_{1}, \cdots, \omega_{n},\left(f_{i}\right)_{1 \leqslant i<\infty}\right.$, $\left.\left(g_{k m}\right)_{1 \leq m<\infty ; 1 \leqq k \leqq n}\right\rangle$ is $\bar{B}$, as we have noted at the end of the proof of Theorem 1 . Let $\bar{D}$ be the algebraic closure of the field of constants $D$ of $\mathcal{F}\left\langle\tilde{t}, \lambda_{1}, \cdots, \lambda_{n}\right\rangle$.

Let $\pi$ be any zero of $L(t, y)$ such that the field of constants of $\mathcal{F}\langle t, \pi\rangle$ is $C$. Let $(t, \pi) \rightarrow(\tilde{t}, \lambda)$ be a generic extension of the specialization $t \rightarrow \tilde{t}$ over $\mathcal{F}$. Then $\lambda=\sum_{j=1}^{n} b_{j} \lambda_{j}$ where each $b_{j}$ is a constant. By Corollary 2 of Lemma 1 we may assume that $b_{j} \in \bar{D}(j=1, \cdots, n)$. If $b$ is any element of $D$ we may write $b=P Q^{-1}$ where $P, Q \in \mathcal{F}\langle\tilde{t}\rangle\left\{\lambda_{1}, \cdots, \lambda_{n}\right\}$; it follows that $b$ may be expanded into a power series in $\beta$, having integral powers a finite number of which are negative, with coefficients belonging to $\mathscr{F}\left\langle\tilde{t}, \omega_{1}, \cdots, \omega_{n},\left(f_{i}\right),\left(g_{k m}\right)\right\rangle$, i.e. with coefficients belonging to $\bar{B}$. Consequently any element of $\bar{D}$ can be expanded into a power series with fractional powers and coefficients belonging to $\bar{B}$. Replacing $\beta$ by a suitable power of itself we may lose no generality in supposing that $b_{1}, \cdots, b_{n}$ may be expanded into power series $b_{j}=\beta^{r_{j}} \sum_{r=0}^{\infty} d_{j i} \beta^{i}$ (each $d_{j i} \in \bar{B}, d_{j 0} \neq 0, r_{j}$ integers). Therefore we may write $\lambda=\sum_{j=1}^{\infty} b_{j} \lambda_{j}$ $=\sum_{j \in J}\left(d_{j 0} \omega_{j}\right) \beta^{r}+\cdots$ where $r=\min \left(r_{1}, \cdots, r_{n}\right)$ and $J$ is the set of all integers $j$ with $1 \leqq j \leqq n$ and $r_{j}=r$. If $r=0$ then $(\tilde{t}, \lambda) \rightarrow\left(\bar{t}, \sum_{j \in J} d_{j 0} \omega_{j}\right)$ is a specialization over $\mathcal{F}$. But there obviously exists a specialization $\left(d_{10}, \cdots, d_{n 0}\right)$ $\rightarrow\left(\bar{d}_{10}, \cdots, \bar{d}_{n 0}\right)$ with $\bar{d}_{j 0} \in C$ and $d_{j 0} \neq 0$, so that $\sum_{j \in J} \bar{d}_{j 0} \omega_{j}=\omega \neq 0$. Therefore $(t, \pi) \rightarrow(\bar{t}, \omega)$ is a specialization over $\mathcal{F}$ and the specialization is analytic if $\bar{B}=C$. If $r>0(t, \pi) \rightarrow(\bar{t}, 0)$ is an analytic specialization over $\mathcal{F}$. If $r<0$ then $\left(t, \pi^{-1}\right) \rightarrow\left(\tilde{t}, \lambda^{-1}\right) \rightarrow(\bar{t}, 0)$ is an analytic specialization over $\mathcal{F}$.

Also, $\lambda^{\prime} \lambda^{-1}=\beta^{-r} \lambda\left(\beta^{-r} \lambda\right)^{-1}$ and since the lowest power of $\beta$ in $\beta^{-r} \lambda$ is zero there exists a nonzero specialization over $\mathcal{F} \beta^{-r} \lambda \rightarrow \omega$, and this specialization is analytic if $\bar{B}=C$. Hence $\left(t, \lambda^{\prime} \lambda^{-1}\right) \rightarrow\left(\bar{t}, \omega^{\prime} \omega^{-1}\right)$ is a specialization, analytic specialization, over $\mathcal{F}$ according as $\bar{B} \supset C$ or $\bar{B}=C$. 
Corollary. Let $t, \bar{t}, \bar{t}, L(t, y)$ be as in Theorem 3 and let $\left(\pi_{1}, \cdots, \pi_{n}\right)$ be a fundamental system of zeros of $L(t, y)$ such that $F\left\langle t, \pi_{1}, \cdots, \pi_{n}\right\rangle$ is a P.V.E. of $\mathcal{F}\langle t\rangle$ with algebraic matric group $G$ which contains the full diagonal group. Then the analytic specialization $t \rightarrow \bar{t}$ over $\mathcal{F}$ can be extended to a specialization $\left(t, \pi_{1}, \cdots, \pi_{n}\right) \rightarrow\left(\bar{t}, \omega_{1}, \cdots, \omega_{n}\right)$ over $\mathcal{F}$ where the field of constants of $\mathcal{F}\left\langle\bar{t}, \omega_{1}, \cdots, \omega_{n}\right\rangle$ is $C$. If the field of constants $B$ of $\mathcal{F}\left\langle\bar{t},\left(f_{i}\right)_{1 \leq i<\infty}\right\rangle$ equals $C$ then the specialization $\left(t, \pi_{1}, \cdots, \pi_{n}\right) \rightarrow\left(\bar{t}, \omega_{1}, \cdots, \omega_{n}\right)$ over $\mathcal{F}$ is analytic.

Proof. By Theorem 3 there exists $\left(\omega_{1}, \cdots, \omega_{n}\right) \omega_{i} \neq 0(i=1, \cdots, n)$ such that $\left(t, \pi_{1}^{\prime} \pi_{1}^{-1}, \cdots, \pi_{n}^{\prime} \pi_{n}^{-1}\right) \rightarrow\left(\bar{t}, \omega_{1}^{\prime} \omega_{1}^{-1}, \cdots, \omega_{n}^{\prime} \omega_{n}^{-1}\right)$ is a specialization over $\mathcal{F}$, and the field of constants of $\mathcal{F}\left\langle\bar{t}, \omega_{1}, \cdots, \omega_{n}\right\rangle$ is $C$. Since $G$ contains the full diagonal group the differential equation of lowest order which $\pi_{i}$ satisfies over $\mathcal{F}\left\langle t, \pi_{1}^{\prime} \pi_{1}^{-1}, \cdots, \pi_{n}^{\prime} \pi_{n}^{-1}, \pi_{1}, \cdots, \pi_{i-1}\right\rangle$ is $y^{\prime}-\pi_{i}^{\prime} \pi_{i}^{-1} y=0$. Since $\omega_{i}$ is a solution of $y^{\prime}-\omega_{i}^{\prime} \omega_{i}^{-1} y=0\left(t, \pi_{1}^{\prime} \pi_{1}^{-1}, \cdots, \pi_{n}^{\prime} \pi_{n}^{-1}, \pi_{1}, \cdots, \pi_{i}\right) \rightarrow\left(\bar{t}, \omega_{1}^{\prime} \omega_{1}^{-1}\right.$, $\left.\cdots, \omega_{n}^{\prime} \omega_{n}^{-1}, \omega_{1}, \cdots, \omega_{i}\right)$ is a specialization over $\mathcal{F}$. If $B=C$ then the specialization $\left(t, \pi_{1}^{\prime} \pi_{1}^{-1}, \cdots, \pi_{n}^{\prime} \pi_{n}^{-1}\right) \rightarrow\left(\bar{t}, \omega_{1}^{\prime} \omega_{n}^{-1}, \cdots, \omega_{n}^{\prime} \omega_{n}^{-1}\right)$ over $\mathcal{F}$ is analytic and by Corollary 2 of Lemma $2\left(t, \pi_{1}, \cdots, \pi_{n}\right) \rightarrow\left(\bar{t}, \omega_{1}, \cdots, \omega_{n}\right)$ is an analytic specialization over $\mathcal{F}$.

This corollary does not say that $\omega_{1}, \cdots, \omega_{n}$ are linearly independent. In fact, as we shall show by example, it may be impossible to find a linearly independent system of solutions $\left(\omega_{1}, \cdots, \omega_{n}\right)$ of $L(\vec{t}, y)$ such that $\left(t, \pi_{1}, \cdots, \pi_{n}\right) \rightarrow\left(\bar{t}, \omega_{1}, \cdots, \omega_{n}\right)$ is a specialization over $\mathcal{F}$. However, if the algebraic matric group $G$ of $\mathcal{F}\left\langle t, \pi_{1}, \cdots, \pi_{n}\right\rangle$ over $\mathcal{F}\langle t\rangle$ contains the full triangular group then we have:

Theorem 4. Let $t, \tilde{t}, \bar{t}, L(t, y)$ be as in Theorem 3 and let $\left(\pi_{1}, \cdots, \pi_{n}\right)$ be a fundamental system of zeros of $L(t, y)$ such that $F\left\langle t, \pi_{1}, \cdots, \pi_{n}\right\rangle$ is a P.V.E. of $\mathcal{F}\langle t\rangle$ with algebraic matric group $G$ which contains the full triangular group. Then there exists a fundamental system of zeros $\left(\omega_{1}, \cdots, \omega_{n}\right)$ of $L(\bar{t}, y)$ such that $\mathcal{F}\left\langle\bar{t}, \omega_{1}, \cdots, \omega_{n}\right\rangle$ is a P.V.E. of $\mathcal{F}\langle\bar{t}\rangle$ and $\left(t, \pi_{1}, \cdots, \pi_{n}\right) \rightarrow\left(\bar{t}, \omega_{1}, \cdots, \omega_{n}\right)$ is $a$ specialization over $\mathcal{F}$. If the field of constants $B$ of $\mathcal{F}\left\langle\bar{t},\left(f_{i}\right)_{1 \leq i<\infty}\right\rangle$ equals $C$ then the specialization $\left(t, \pi_{1}, \cdots, \pi_{n}\right) \rightarrow\left(\bar{t}, \omega_{1}, \cdots, \omega_{n}\right)$ over $\mathcal{F}$ is analytic.

Proof. We use induction on $n$ to prove the existence of a fundamental system of zeros $\left(\alpha_{1}, \cdots, \alpha_{n}\right)$ of $L(\bar{t}, y)$ such that the field of constants of $\mathcal{F}\left\langle\bar{t}, \alpha_{1}, \cdots, \alpha_{n}\right\rangle$ belongs to $\bar{B}$, the algebraic closure of $B$, and $\left(t, \pi_{1}, \cdots, \pi_{n}\right)$ $\rightarrow\left(\bar{t}, \alpha_{1}, \cdots, \alpha_{n}\right)$ is an analytic specialization over $\mathcal{F}$. For $n=1$ our assertion is valid for by Theorem 3 there exists $\lambda=\beta^{r} \sum_{i=0}^{\infty} g_{i} \beta^{i}$ such that $\left(t, \pi_{1}\right) \rightarrow(\tilde{t}, \lambda)$ is a generic specialization over $\mathcal{F}$. Since $G$ contains the full triangular group any constant multiple of $\lambda$ is a generic specialization of $\lambda$ over $\mathcal{F}\langle t\rangle$, so that $\left(t, \pi_{1}\right) \rightarrow\left(\tilde{t}, \sum_{i=0}^{\infty} g_{i} \beta^{i}\right)$ is a generic specialization over $\mathcal{F}$ and $\left(t, \pi_{1}\right) \rightarrow\left(\bar{t}, g_{0}\right) g_{0} \neq 0$ is an analytic specialization over $\mathcal{F}$. Let $n>1$ and let our assertion be true for lower values than $n$. Let $L_{1}\left(t, \pi_{1}, y\right)$ be the homogeneous linear differential polynomial of order $n-1$ in $y$ which has $\left(\left(\pi_{2} \pi_{1}^{-1}\right), \cdots,\left(\pi_{n} \pi_{1}^{-1}\right)\right)$ as a fundamental system of zeros; then $L_{1}\left(t, \pi_{1}, y\right)=a_{0}(t) \pi_{1} y^{(n-1)}+\cdots$. Since $a_{0}(\bar{t}) g_{0} \neq 0$ 
and $\left(t, \pi_{1}\right) \rightarrow\left(\bar{t}, g_{0}\right)$ is an analytic specialization over $\mathcal{F}$, by our induction hypothesis there exists a fundamental system of zeros $\left(\mu_{2}, \cdots, \mu_{n}\right)$ of $L_{1}\left(\bar{t}, g_{0}, y\right)$ such that

$$
\left(t, \pi_{1},\left(\pi_{2} \pi_{1}^{-1}\right)^{\prime}, \cdots,\left(\pi_{n} \pi_{1}^{-1}\right)^{\prime}\right) \rightarrow\left(\bar{t}, g_{0}, \mu_{2}, \cdots, \mu_{n}\right)
$$

is an analytic specialization over $\mathcal{F}$ and the field of constants of

$$
\mathfrak{F}\left\langle\bar{t}, g_{0}, \mu_{2}, \cdots, \mu_{n}\right\rangle
$$

belongs to $\bar{B}$. For the group of $\mathcal{F}\left\langle t, \pi_{1},\left(\pi_{2} \pi_{1}^{-1}\right)^{\prime}, \cdots,\left(\pi_{n} \pi_{1}^{-1}\right)^{\prime}\right\rangle$ over $\mathcal{F}\left\langle t, \pi_{1}\right\rangle$ contains the full triangular group. Now the equation of lowest order that $\pi_{i} \pi_{1}^{-1}$ satisfies over

$$
\mathcal{F}\left\langle t, \pi_{1}, \cdots, \pi_{i-1},\left(\pi_{i} \pi_{1}^{-1}\right)^{\prime}, \cdots,\left(\pi_{n} \pi_{1}^{-1}\right)^{\prime}\right\rangle
$$

is $y^{\prime}-\left(\pi_{i} \pi_{1}^{-1}\right)^{\prime}=0$. Hence the analytic specialization

$$
\left(t, \pi_{1},\left(\pi_{2} \pi_{1}^{-1}\right)^{\prime}, \cdots,\left(\pi_{n} \pi_{1}^{-1}\right)^{\prime}\right) \rightarrow\left(\bar{t}, g_{0}, \mu_{2}, \cdots, \mu_{n}\right)
$$

over $\mathcal{F}$ can be successively extended to $\pi_{i} \pi_{1}^{-1} \rightarrow \theta_{i}$ where $\theta_{i}$ is a nonzero solution of $y^{\prime}-\mu_{i}=0(i=2, \cdots, n)$ such that the field of constants of $F\left\langle\bar{t}, g_{0}, \theta_{2}, \cdots\right.$, $\left.\theta_{n}\right\rangle$ belongs to $\bar{B}$. Let $\alpha_{1}=g_{0} \alpha_{i}=g_{0} \theta_{i}(i=2, \cdots, n)$ then $\left(t, \pi_{1}, \cdots, \pi_{n}\right)$ $\rightarrow\left(\bar{t}, \alpha_{1}, \cdots, \alpha_{n}\right)$ is an analytic specialization over $\mathcal{F}$. Also $W\left(\alpha_{1}, \cdots, \alpha_{n}\right) \neq 0$; for suppose there exist constants $a_{i}$ such that $\sum_{i=1}^{n} a_{i} \alpha_{i}=0$. Since $\alpha_{i} \neq 0$ $(1 \leqq i \leqq n)$ at least two of the elements $a_{i}$ are not zero. Dividing through by $\alpha_{1}$ we get $a_{1}+\sum_{i=2}^{n} a_{i}\left(\alpha_{i} \alpha_{1}^{-1}\right)=0$, so that $\sum_{i=2}^{n} a_{i}\left(\alpha_{i} \alpha_{1}^{-1}\right)=\sum_{i=2}^{n} a_{i} \mu_{i}=0$ with at least one of the constants $a_{i}$ different from zero, contradicting our induction assumption. Hence $W\left(\alpha_{1}, \cdots, \alpha_{n}\right) \neq 0$ and our assertion is proved.

Now let $\left(\sigma_{1}, \cdots, \sigma_{n}\right)$ be a fundamental system of zeros of $L(\bar{t}, y)$ such that the field of constants of $\mathcal{F}\left\langle\bar{t}, \sigma_{1}, \cdots, \sigma_{n}\right\rangle$ is $C$. Then $\sigma_{i}=\sum_{j=1}^{n} b_{i j} \alpha_{j}$ and we may assume each $b_{i j} \in \bar{B}$ (Corollary 2, Lemma 1). Let $\left(a_{i j}\right)=\left(b_{i j}\right)^{-1}$ then $\alpha_{i}=\sum_{j=1}^{n} a_{i j} \sigma_{j}$ with each $a_{i j} \in \bar{B}$; there obviously exists a specialization, over $\mathcal{F}\langle\bar{t}\rangle, \quad\left(a_{i j}\right) \rightarrow\left(\bar{a}_{i j}\right)$ with each $\bar{a}_{i j} \in C$ such that determinant $\left(\bar{a}_{i j}\right) \neq 0$. Let $\omega_{i}=\sum_{j=1}^{n} \bar{a}_{i j} \sigma_{j}$ then $\left(t, \pi_{1}, \cdots, \pi_{n}\right) \rightarrow\left(\bar{t}, \omega_{1}, \cdots, \omega_{n}\right)$ is a specialization over $\mathcal{F}$, and the field of constants of $\mathcal{F}\left\langle\bar{t}, \omega_{1}, \cdots, \omega_{n}\right\rangle$ is $C$.

The examples below show that if the group of $\mathcal{F}\left\langle t, \pi_{1}, \cdots, \pi_{n}\right\rangle$ over $\mathscr{F}\langle t\rangle$ does not contain the full triangular group there may not exist a specialization $\left(t, \pi_{1}, \cdots, \pi_{n}\right) \rightarrow\left(\bar{t}, \omega_{1}, \cdots, \omega_{n}\right)$ over $\mathcal{F}$ such that $\mathcal{F}\left\langle\bar{t}, \omega_{1}, \cdots, \omega_{n}\right\rangle$ is a P.V.E. of $\mathcal{F}\langle\bar{t}\rangle$.

EXAMPLE 1. Let $\mathcal{F}$ be the differential field of rational functions of $x\left(x^{\prime}=1\right)$ over the complex numbers. Let $t=(\log x)^{-1}$ then the differential equation of lowest order that $t$ satisfies over $\mathcal{F}$ is $x y^{\prime}+y^{2}=0$. Now, $t \rightarrow 0$ is an analytic specialization over $\mathcal{F}$, for $t \rightarrow 0+\sum_{i=0}^{\infty}(-1)^{i}(\log x)^{i} \beta^{i+1}$ is a generic specialization over $\mathcal{F}$, since $\sum_{i=0}^{\infty}(-\log x)^{i} \beta^{i+1}=\beta[1+(\log x) \beta]^{-1}$, which is not algebraic over $\mathcal{F}$, is a solution of $x y^{\prime}+y^{2}=0$. Let $L(t, y)=x y^{\prime \prime}+y^{\prime}$; then $\log x$ is a zero of $L(t, y)$ and the specialization $t \rightarrow 0$ can not be extended to a specialization of $(t, \log x)$ over $\mathfrak{F}$. 
EXAmple 2. Let $\mathcal{F}$ be the field of complex numbers, let $t=e^{x}, \bar{t}=0$ and let $L(t, y)=y^{\prime \prime}-\left[\left(1+2^{1 / 2}\right) e^{x}+1\right] y^{\prime}+2^{1 / 2} e^{2 x} y . L(t, y)$ has a fundamental system of zeros $\left(e^{e^{x}}, e^{2^{1 / 2} e^{x}}\right)$. As we have shown above in Example 2 of Theorem 2, the specialization $t \rightarrow \bar{t}$ over $\mathcal{F}$ is analytic and the algebraic matric group of $\mathcal{F}\left\langle e^{x}, e^{e^{x}}, e^{2^{1 / 2} e^{x}}\right\rangle$ over $\mathcal{F}\langle t\rangle$ is the full diagonal group. Now, $L(\bar{t}, y)=y^{\prime \prime}-y^{\prime}$ which has a fundamental system of zeros $\left(1, e^{x}\right)$; but the specialization $t \rightarrow 0$ has only one possible extension $\left(t, e^{e^{x}}, e^{2^{1 / 2} e^{x}}\right) \rightarrow\left(0, c_{1}, c_{2}\right)$ where $c_{1}, c_{2}$ are constants which do not give a fundamental system of zeros of $L(\bar{t}, y)$.

Lemma 3. Let $t=\left(t_{1}, \cdots, t_{r}\right)$ be differential indeterminates over $\mathcal{F}$ and let $\pi$ be a nonzero solution of $a_{0}(t) y^{\prime}+a_{1}(t) y=0\left(a_{0}(t), a_{1}(t) \in \mathcal{F}\{t\}\right.$ without common divisors) such that $\mathfrak{F}\langle t, \pi\rangle$ is a P.V.E. of $\mathcal{F}\langle t\rangle$. Then any specialization $t \rightarrow \bar{t}$ such that $a_{0}(\bar{t}) \neq 0$ can be extended to a specialization $(t, \pi) \rightarrow(\bar{t}, \bar{\pi})$ over $\mathcal{F}$ such that $\bar{\pi} \neq 0$ and $\mathcal{F}\langle\bar{t}, \bar{\pi}\rangle$ is a P.V.E. of $\mathcal{F}\langle\bar{t}\rangle$.

Proof. If $\pi$ is not algebraic over $\mathscr{F}\langle t\rangle$ then any nonzero solution $\tilde{\pi}$ of $a_{0}(\bar{t}) y^{\prime}-a_{1}(\bar{t}) y=0$ such that $\mathcal{F}\langle\bar{t}, \bar{\pi}\rangle$ is a P.V.E. of $\mathcal{F}\langle\bar{t}\rangle$ will do. Suppose $\pi$ is algebraic over $\mathscr{F}\langle t\rangle$; then since $\pi$ satisfies a h.l.d. equation of order 1 over $\mathcal{F}\langle t\rangle$ any automorphism of $\mathcal{F}\langle t, \pi\rangle$ over $\mathscr{F}\langle t\rangle$ takes $\pi$ into $c \pi c \in C$. Also, the group of automorphisms of $\mathcal{F}\langle t, \pi\rangle$ over $\mathcal{F}\langle t\rangle$ is finite of order $k$ so that $c^{k}=1$ and

$$
\pi^{k}=P(t) / Q(t)
$$

$(P(t), Q(t) \in \mathcal{F}\{t\}$ without common divisors; $k$ an integer) and

$$
a_{1}(t) / a_{0}(t)=\frac{P(t)^{\prime} Q(t)-P(t) Q(t)^{\prime}}{k P(t) Q(t)}
$$

so that $a_{0}\left(Q P^{\prime}-P Q^{\prime}\right)=k a_{1} P Q$. Assume $P(\bar{t})=0$ and let $R$ be an irreducible factor of $P$ such that $R(\bar{t})=0$. Let $P=R^{n} S(n>0, S$ not divisible by $R)$. Then $R$ does not divide $a_{0}$ or $Q$ so that $R^{n}$ divides

$$
Q P^{\prime}-P Q^{\prime}=Q\left(n R^{n-1} R^{\prime} S+R^{n} S^{\prime}\right)-R^{n} S Q^{\prime} .
$$

Hence $R$ divides $Q R^{\prime} S$; it follows that $R$ divides $R^{\prime}$ which is impossible since $R^{\prime}$ is of the same degree as $R$ but is of higher order. Hence $P(\bar{t}) \neq 0$, and for the same reason $Q(\bar{t}) \neq 0$ so that any solution $\bar{\pi}$ of $Q(\bar{t}) y^{k}-P(\bar{t})=0$ has the property that $(t, \pi) \rightarrow(\bar{t}, \bar{\pi})$ is a specialization over $\mathcal{F}$.

Theorem 5. Let $t=\left(t_{1}, \cdots, t_{r}\right)$ be differential indeterminates over $\mathcal{F}$, let $L(t, y)=a_{0}(t) y^{(n)}+\cdots+a_{n}(t) y \in \mathcal{F}\{t, y\}$ and let $\left(\pi_{1}, \cdots, \pi_{n}\right)$ be a fundamental system of zeros of $L(t, y)$ such that $\mathscr{F}\left\langle t, \pi_{1}, \cdots, \pi_{n}\right\rangle$ is a P.V.E. of $\mathcal{F}\langle t\rangle$ with algebraic matric group $G$ containing the unimodular group. Then any specialization $t \rightarrow \bar{t}$ over $\mathcal{F}$ such that $a_{0}(\bar{t}) \neq 0$ can be extended to a specialization $\left(t, \pi_{1}, \cdots, \pi_{n}\right) \rightarrow\left(\bar{t}, \bar{\pi}_{1}, \cdots, \bar{\pi}_{n}\right)$ over $\mathcal{F}$ such that $\mathcal{F}\left\langle\bar{t}, \bar{\pi}_{1}, \cdots, \bar{\pi}_{n}\right\rangle$ is a P.V.E. of $\mathcal{F}\langle\bar{t}\rangle$ and the Wronskian $W\left(\bar{\pi}_{1}, \cdots, \bar{\pi}_{n}\right) \neq 0$. 
Proof. If the dimension of $G$ is $n^{2}$ then any fundamental system of zeros $\left(\bar{\pi}_{1}, \cdots, \bar{\pi}_{n}\right)$ of $L(\bar{t}, y)$ such that $\mathscr{F}\left\langle\bar{t}, \bar{\pi}_{1}, \cdots, \bar{\pi}_{n}\right\rangle$ is a P.V.E. of $F\langle\bar{t}\rangle$ will do. Let the dimension of $G$ be $n^{2}-1$. By Lemma 3 the specialization $t \rightarrow \bar{t}$ over $\mathcal{F}$ can be extended to $(t, W) \rightarrow(\bar{t}, \bar{W})$ where $W=W\left(\pi_{1}, \cdots, \pi_{n}\right), \bar{W} \neq 0$ and the field of constants of $\mathcal{F}\langle\bar{t}, \bar{W}\rangle$ is $C$; for $W$ is a zero of $a_{0}(t) y^{\prime}-a_{1}(t) y$. Now, the group of $\mathscr{F}\left\langle t, \pi_{1}, \cdots, \pi_{n}\right\rangle$ over $\mathfrak{F}\langle t, W\rangle$ is the unimodular group of dimension $n^{2}-1$ which equals degree of transcendence of $\mathfrak{F}\left\langle t, \pi_{1}, \cdots, \pi_{n}\right\rangle$ over $\mathcal{F}\langle t, W\rangle$. Hence the differential equation of lowest order that $\pi_{i}$ satisfies over $\dot{\mathcal{F}}\left\langle t, W, \pi_{1}, \cdots, \pi_{i-1}\right\rangle,(i=1, \cdots, n-1)$, is $L(t, y)=0$. For otherwise the sum of the orders would be less than $n^{2}-1$. Since $\pi_{n}$ satisfies an equation of order $n-1$, i.e. $W\left(\pi_{1}, \cdots, \pi_{n-1}, y\right)=W\left(\pi_{1}, \cdots, \pi_{n}\right)$. Therefore any $n-1$ linearly independent zeros $\left(\bar{\pi}_{1}, \cdots, \bar{\pi}_{n-1}\right)$ of $L(t, y)$, such that the field of constants of $\mathcal{F}\left\langle\bar{t}, \bar{\pi}_{1}, \cdots, \bar{\pi}_{n-1}\right\rangle$ is $C$, will do. The differential equation of lowest order that $\pi_{n}$ satisfies over $\mathcal{F}\left\langle t, W, \pi_{1}, \cdots, \pi_{n-1}\right\rangle$ is $W\left(\pi_{1}, \cdots, \pi_{n-1}, y\right)$ $-W=0$ which is linear and of order $n-1$. The coefficient of $y^{(n-1)}$ is $W\left(\pi_{1}, \cdots, \pi_{n-1}\right)$. Since $W\left(\bar{\pi}_{1}, \cdots, \bar{\pi}_{n-1}\right) \neq 0$ any nonzero solution $\bar{\pi}_{n}$ of $W\left(\bar{\pi}_{1}, \cdots, \bar{\pi}_{n-1}, y\right)-\bar{W}=0$ such that $\mathcal{F}\left\langle\bar{t}, \bar{\pi}_{1}, \cdots, \bar{\pi}_{n}\right\rangle$ is a P.V.E. of $\mathcal{F}\langle\bar{t}\rangle$ has the property that $\left(t, W, \pi_{1}, \cdots, \pi_{n}\right) \rightarrow\left(\bar{t}, \bar{W}, \bar{\pi}_{1}, \cdots, \bar{\pi}_{n}\right)$ is a specialization over $\mathcal{F}$.

\section{Generic equation with group $G$}

1. Definition. Let $G$ be an $n \times n$ algebraic matric group and let $L(t, y)$ $=Q_{0}\left(t_{1}, \cdots, t_{r}\right) y^{(n)}+\cdots+Q_{n}\left(t_{1}, \cdots, t_{r}\right) y \in C\left\{t_{1}, \cdots, t_{r}, y\right\}$. Let $\left(\pi_{1}, \cdots, \pi_{n}\right)$ be a fundamental system of zeros of $L(t, y)$ such that $C\left\langle t_{1}, \cdots, t_{r}, \pi_{1}, \cdots, \pi_{n}\right\rangle$ is a P.V.E. of $C\left\langle t_{1}, \cdots, t_{r}\right\rangle$ with group $G$. Then $L(t, y)=0$ will be called a "generic equation with group $G$ " if:

(1) $t_{1}, \cdots, t_{r}$ are differentially algebraically independent over $C$, and $C\left\langle t_{1}, \cdots, t_{r}\right\rangle \subset C\left\langle\pi_{1}, \cdots, \pi_{n}\right\rangle$.

(2) For every specialization $\left(t_{1}, \cdots, t_{r}, \pi_{1}, \cdots, \pi_{n}\right) \rightarrow\left(\bar{t}_{1}, \cdots, \bar{t}_{r}\right.$, $\left.\bar{\pi}_{1}, \cdots, \bar{\pi}_{n}\right)$ over $C$ such that $C\left\langle\bar{t}_{1}, \cdots, \bar{t}_{r}, \bar{\pi}_{1}, \cdots, \bar{\pi}_{n}\right\rangle$ is a P.V.E. of $C\left\langle\bar{t}_{1}, \cdots, \bar{t}_{r}\right\rangle$ and field of constants of $C\left\langle\bar{t}_{1}, \cdots, \bar{t}_{r}\right\rangle$ is $C$, the algebraic matric group $H$ of this extension corresponding to the fundamental system of zeros $\left(\bar{\pi}_{1}, \cdots, \bar{\pi}_{n}\right)$ of $L(\bar{t}, y)$ is a subgroup of $G$.

(3) If $\left(\omega_{1}, \cdots, \omega_{n}\right)$ is a fundamental system of zeros of $L(y)=y^{(n)}$ $+a_{1} y^{(n-1)}+\cdots+a_{n} y \in \mathcal{F}\{y\}$ where $\mathcal{F}$ is any differential field with field of constants $C$, and $\mathcal{F}\left\langle\omega_{1}, \cdots, \omega_{n}\right\rangle$ is a P.V.E. of $\mathcal{F}$ with algebraic matric group $H \subseteq G$, then there exists a specialization $\left(t_{1}, \cdots, t_{r}\right) \rightarrow\left(\bar{t}_{1}, \cdots, \bar{t}_{r}\right)$ over $F$ with $\bar{t}_{i} \in \mathcal{F}$ such that $Q_{0}\left(\bar{t}_{1}, \cdots, \bar{t}_{r}\right) \neq 0$ and

$$
a_{i}=Q_{i}\left(\bar{t}_{1}, \cdots, \bar{t}_{r}\right) Q_{0}^{-1}\left(\bar{t}_{1}, \cdots, \bar{t}_{r}\right) .
$$

\section{Necessary and sufficient conditions.}

Lemma 1. Let $G$ be an $n \times n$ algebraic matric group and let $L(t, y)$ $=Q_{0}\left(t_{1}, \cdots, t_{r}\right) y^{(n)}+\cdots+Q_{n}\left(t_{1}, \cdots, t_{r}\right) y \in C\left\{t_{1}, \cdots, t_{r}, y\right\}$ be a "generic 
equation with group G." Then $r=n$.

Proof. By (1) $C\left\langle t_{1}, \cdots, t_{r}\right\rangle \subseteq C\left\langle\pi_{1}, \cdots, \pi_{n}\right\rangle$ so that $r \leqq n$. Suppose $r<n$. Let $y_{1}, \cdots, y_{n}$ be $n$ differential indeterminates over $C$. Then $C\left\langle y_{1}, \cdots, y_{n}\right\rangle$ is a P.V.E. of $C\left\langle P_{1}\left(y_{1}, \cdots, y_{n}\right), \cdots, P_{n}\left(y_{1}, \cdots, y_{n}\right)\right\rangle$ where

$$
\begin{aligned}
& P_{i}\left(y_{1}, \cdots, y_{n}\right)=\frac{W_{i}\left(y_{1}, \cdots, y_{n}\right)}{W_{0}\left(y_{1}, \cdots, y_{n}\right)} \quad(i=1, \cdots, n), \\
& W_{i}=(-1)^{i}\left|\begin{array}{lll}
y_{1} & \cdots & y_{n} \\
\vdots & & \\
y_{1}^{(n-i-1)} & y_{n}^{(n-i-1)} \\
y_{1}^{(n-i+1)} & y_{n}^{(n-i+1)} \\
\vdots & \vdots \\
y_{1}^{(n)} & y_{n}^{(n)}
\end{array}\right| .
\end{aligned}
$$

Let $g$ be the differential field of invariants of $G$ in $C\left\langle y_{1}, \cdots, y_{n}\right\rangle$. Then $C\left\langle y_{1}, \cdots, y_{n}\right\rangle$ is a P.V.E. of $G$ with group $G$, for $C\left\langle P_{1}, \cdots, P_{n}\right\rangle \subset \mathcal{G}$. Since the degree of differential transcendency of $C\left\langle P_{1}, \cdots, P_{n}\right\rangle$ over $C$ is $n$ there can not exist any specialization $\left(t_{1}, \cdots, t_{r}\right) \rightarrow\left(\bar{t}_{1}, \cdots, \bar{t}_{r}\right)$ over $C$ such that

$$
P_{i}=\frac{Q_{i}\left(\bar{t}_{1}, \cdots, \bar{t}_{r}\right)}{Q_{0}\left(\bar{t}_{1}, \cdots, \bar{t}_{r}\right)}
$$

violating (3). Hence $r=n$.

This lemma shows that if an $n \times n$ algebraic matric group $G$ has a "generic equation with group $G$ " then it is necessary that the differential field of invariants of $G$ in $C\left\langle y_{1}, \cdots, y_{n}\right\rangle$ be purely differentially transcendental over $C$.

Lemma 2. Let $G$ be an $n \times n$ algebraic matric group over $C$; let

$$
C\left\langle t_{1}\left(y_{1}, \cdots, y_{n}\right), \cdots, t_{n}\left(y_{1}, \cdots, y_{n}\right)\right\rangle
$$

be the field of invariants of $G$ in $C\left\langle y_{1}, \cdots, y_{n}\right\rangle$, where $y_{1}, \cdots, y_{n}$ are $n$ differential indeterminates over $C$. Let

$$
\begin{aligned}
t_{i}\left(y_{1}, \cdots, y_{n}\right) & =\frac{f_{i}\left(y_{1}, \cdots, y_{n}\right)}{g_{i}\left(y_{i}, \cdots, y_{n}\right)} f_{i}, g_{i} \in C\left\{y_{1}, \cdots, y_{n}\right\} \quad(i=1, \cdots, n), \\
P_{i}\left(y_{1}, \cdots, y_{n}\right) & =\frac{Q_{i}\left(t_{1}, \cdots, t_{n}\right)}{Q_{0}\left(t_{1}, \cdots, t_{n}\right)}
\end{aligned}
$$

where $P_{i}\left(y_{1}, \cdots, y_{n}\right)$ is given by $(\mathrm{A})$. Let

$$
Q_{0}\left(t_{1}, \cdots, t_{n}\right)=\frac{R\left(f_{1}, \cdots, f_{n}, g_{1}, \cdots, g_{n}\right)}{\prod_{i=1}^{n} g_{i}^{d_{i}}\left(y_{1}, \cdots, y_{n}\right)}=\frac{R^{*}\left(y_{1}, \cdots, y_{n}\right)}{\prod g_{i} d_{i}\left(y_{1}, \cdots, y_{n}\right)} .
$$


Let $W_{0}\left(y_{1}, \cdots, y_{n}\right) \in\left\{R^{*}\left(y_{1}, \cdots, y_{n}\right) \prod_{i=1}^{n} g_{i}\left(y_{1}, \ldots, y_{n}\right)\right\}$ and let $\mathcal{F}\left\langle\omega_{1}, \cdots, \omega_{n}\right\rangle$ be a P.V.E. of $\mathcal{F}$ with group $H \subseteq G$ where $\left(\omega_{1}, \cdots, \omega_{n}\right)$ is a fundamental system of zeros of

$$
L(y)=y^{(n)}+a_{1} y^{(n-1)}+\cdots+a_{n} y \in F\{y\} .
$$

Then there exists a specialization $\left(t_{1}, \cdots, t_{n}\right) \rightarrow\left(\bar{t}_{1}, \cdots, \bar{t}_{n}\right)$ over $C$ with $\bar{t}_{i} \in \mathcal{F}$ such that

$$
a_{i}=\frac{Q_{i}\left(\bar{t}_{1}, \cdots, \bar{t}_{n}\right)}{Q_{0}\left(\bar{t}_{1}, \cdots, \bar{t}_{n}\right)}
$$

Proof. Since

$$
W_{0}\left(\omega_{1}, \cdots, \omega_{n}\right) \neq 0, \quad R^{*}\left(\omega_{1}, \cdots, \omega_{n}\right) \prod_{i=1}^{n} g_{i}\left(\omega_{1}, \cdots, \omega_{n}\right) \neq 0 .
$$

Hence

$$
t_{i}\left(\omega_{1}, \cdots, \omega_{n}\right), \quad \frac{Q_{i}\left(t_{1}\left(\omega_{1}, \cdots, \omega_{n}\right), \cdots, t_{n}\left(\omega_{1}, \cdots, \omega_{n}\right)\right.}{Q_{0}\left(t_{1}\left(\omega_{1}, \cdots, \omega_{n}\right), \cdots, t_{n}\left(\omega_{1}, \cdots, \omega_{n}\right)\right.}
$$

are defined. Furthermore $t_{i}\left(\omega_{1}, \cdots, \omega_{n}\right)$ are left invariant by $H$ since $H \subseteq G$, so that $t_{i}\left(\omega_{1}, \cdots, \omega_{n}\right) \in \mathcal{F}$. Also, we have

$$
a_{i}=P_{i}\left(\omega_{1}, \cdots, \omega_{n}\right)=\frac{Q_{i}\left(t_{1}\left(\omega_{1}, \cdots, \omega_{n}\right), \cdots, t_{n}\left(\omega_{1}, \cdots, \omega_{n}\right)\right)}{Q_{0}\left(t_{1}\left(\omega_{1}, \cdots, \omega_{n}\right), \cdots, t_{n}\left(\omega_{1}, \cdots, \omega_{n}\right)\right)} .
$$

Hence the specialization $\left(t_{1}, \cdots, t_{n}\right) \rightarrow\left(\bar{t}_{1}, \cdots, \bar{t}_{n}\right)=\left(t_{1}\left(\omega_{1}, \cdots, \omega_{n}\right), \cdots\right.$, $\left.t_{n}\left(\omega_{1}, \cdots, \omega_{n}\right)\right)$ over $C$ gives us

$$
a_{i}=\frac{Q_{i}\left(\bar{t}_{1}, \cdots, \bar{t}_{n}\right)}{Q_{0}\left(\bar{t}_{1}, \cdots, \bar{t}_{n}\right)}
$$

with $\bar{t}_{i} \in \mathcal{F}$.

We are going to show how to construct a "generic equation with group $G$ " for the following groups $G$ :

(1) The full linear group;

(2) the unimodular group;

(3) the reducible group consisting of all nonsingular matrices $\left(a_{i j}\right) i, j$ $=1, \cdots, n$, such that $a_{r+k, m}=0(k=1, \cdots, s ; m=1, \cdots, r) r, s$ being fixed with $r+s=n$;

(4) the orthogonal group;

(5) the symplectic group.

Our procedure will be as follows. For the differential field $C\left\langle y_{1}, \cdots, y_{n}\right\rangle$, where $y_{1}, \cdots, y_{n}$ are differential indeterminates over $C$, we shall find $n$ differentially algebraically independent generators $t_{1}, \cdots, t_{n}$ over $C$ of the differential field of invariants of $G$ in $C\left\langle y_{1}, \cdots, y_{n}\right\rangle$. We shall then show how 
to obtain $n+1$ differential polynomials $Q_{0}\left(t_{1}, \cdots, t_{n}\right), \cdots, Q_{n}\left(t_{1}, \cdots, t_{n}\right)$ such that

$$
P_{i}\left(y_{1}, \cdots, y_{n}\right)=\frac{Q_{i}\left(t_{1}, \cdots, t_{n}\right)}{Q_{0}\left(t_{1}, \cdots, t_{n}\right)} \quad(i=1, \cdots, n)
$$

where $P_{i}\left(y_{1}, \cdots, y_{n}\right)$ is given by (A). Then

$$
L(t, y)=Q_{0}\left(t_{1}, \cdots, t_{n}\right) y^{(n)}+\cdots+Q_{n}\left(t_{1}, \cdots, t_{n}\right) y=0
$$

will be our "generic equation with group $G$. ."

3. The full linear group. For the full linear group we let $t_{i}=P_{i}\left(y_{1}, \cdots, y_{n}\right)$ and

$$
L(t, y)=y^{(n)}+P_{1}\left(y_{1}, \cdots, y_{n}\right) y^{(n-1)}+\cdots+P_{n}\left(y_{1}, \cdots, y_{n}\right) y .
$$

Conditions (1), (2) and (3) are obviously satisfied.

4. The unimodular group. Let $G$ be the unimodular group. Then the differential subfield $\mathcal{G}$ of $C\left\langle y_{1}, \cdots, y_{n}\right\rangle$ which is left invariant by $G$ is $C\left\langle t_{1}, \cdots, t_{n}\right\rangle$ where $t_{1}=W_{0}\left(y_{1}, \cdots, y_{n}\right)$ and $t_{i}=W_{i}\left(y_{1}, \cdots, y_{n}\right)(i=2, \cdots$. $n), W_{i}\left(y_{1}, \cdots, y_{n}\right)$ being given by (A). For, $W_{i}\left(y_{1}, \cdots, y_{n}\right)$ is left invariant by $G$ and is not left invariant by any other nonsingular linear transformation. Also,

$$
\begin{aligned}
& P_{i}\left(y_{1}, \cdots, y_{n}\right)=\frac{W_{i}\left(y_{1}, \cdots, y_{n}\right)}{W_{0}\left(y_{1}, \cdots, y_{n}\right)}=t_{i} t_{1}^{-1} \quad(i=2, \cdots, n), \\
& P_{1}\left(y_{1}, \cdots, y_{n}\right)=\frac{W_{0}^{\prime}\left(y_{1}, \cdots, y_{n}\right)}{W_{0}\left(y_{1}, \cdots, y_{n}\right)}=t_{1}^{\prime} t_{1}^{-1} .
\end{aligned}
$$

Hence $C\left\langle P_{1}, \cdots, \quad P_{n}\right\rangle \subset C\left\langle t_{1}, \cdots, t_{n}\right\rangle \subset C\left\langle y_{1}, \cdots, y_{n}\right\rangle$. Therefore $\mathcal{G}$ $=C\left\langle t_{1}, \cdots, t_{n}\right\rangle$. Now, let

and let

$$
L(t, y)=t_{1} y^{(n)}-t_{1}^{1} y^{(n-1)}+\sum_{i=2}^{n} t_{i} y^{(n-1)},
$$

$$
\left(t_{1}, \cdots, t_{n}, y_{1}, \cdots, y_{n}\right) \rightarrow\left(\bar{t}_{1}, \cdots, \bar{t}_{n}, \bar{y}_{1}, \cdots, \bar{y}_{n}\right)
$$

be a specialization over $C$ such that $C\left\langle\bar{t}_{1}, \cdots, \bar{t}_{n}, \bar{y}_{1}, \cdots, \bar{y}_{n}\right\rangle$ is a P.V.E. of $C\left\langle\bar{t}_{1}, \cdots, \bar{t}_{n}\right\rangle$. Let $H$ be the algebraic matric group of $C\left\langle\bar{t}_{1}, \cdots, \bar{t}_{n}, \bar{y}_{1}, \cdots, \bar{y}_{n}\right\rangle$ over $C\left\langle\bar{t}_{1}, \cdots, \bar{t}_{n}\right\rangle$ and let $\sigma=\left(a_{i j}\right) \in H$. Then $\bar{t}_{1}=\sigma \bar{t}_{1}=\operatorname{det} .\left(a_{i j}\right) \bar{t}_{1}$, and since $\bar{t}_{1}=W_{0}\left(\bar{y}_{1}, \cdots, \bar{y}_{n}\right) \neq 0$, det $\left(a_{i j}\right)=1$ and $H$ is a subgroup of the unimodular group. Furthermore since $L(t, y)$ satisfies the conditions of Lemma $2 L(t, y)$ $=0$ is a "generic equation with group $G$."

\section{The reducible group.}

TheOREM 1. Let $r, s$ be natural numbers such that $r+s=n$, and let $G$ be the reducible group consisting of all nonsingular matrices $\left(a_{i j}\right)(i, j=1, \cdots, n)$ 
such that $a_{r+k, m}=0(k=1, \cdots, s ; m=1, \cdots, r)$. Then the differential field $\mathrm{G}$ of invariants of $G$ in $C\left\langle y_{1}, \cdots, y_{n}\right\rangle$ is purely differentially transcendental over $C$, and $\mathrm{G}=C\left\langle t_{1}, \cdots, t_{n}\right\rangle$ where

$$
\begin{array}{rlrl}
t_{i} & =\frac{W_{i}\left(y_{1}, \cdots, y_{r}\right)}{W_{0}\left(y_{1}, \cdots, y_{r}\right)} \\
t_{r+i} & =\frac{W_{i}\left(y_{1}, \cdots, y_{n}\right)}{W_{0}\left(y_{1}, \cdots, y_{n}\right)} & (i=1, \cdots, r),
\end{array}
$$

( $W_{i}$ is defined by $\left.(\mathrm{A})\right)$.

Proof. $C\left\langle t_{1}, \cdots, t_{n}\right\rangle$ is, obviously, left invariant by $G$. Also, any nonsingular matrix $\sigma \notin G$ will not leave any of the $t_{i}(i=1, \cdots, r)$ invariant. For, the $t_{i}(i=1, \cdots, r)$ involve only $y_{1}, \cdots, y_{r}$ and if $\sigma \notin G \sigma t_{i}$ must contain at least one $y_{j}(j \neq 1, \cdots, r)$. Since $y_{1}, \cdots, y_{n}$ are differential indeterminates over $C$ they can not satisfy the relation $\sigma t_{i}=t_{i}(i=1, \cdots, r)$.

It remains to show that $C\left\langle P_{1}, \cdots, P_{n}\right\rangle \subset C\left\langle t_{1}, \cdots, t_{n}\right\rangle$. Since $G$ is reducible the differential polynomial $L(y)=y^{(n)}+P_{1}\left(y_{1}, \cdots, y_{n}\right) y^{(n-1)}+\cdots$ $+P_{n}\left(y_{1}, \cdots, y_{n}\right) y$ is linearly reducible over $\mathcal{G}$ (Kolchin [2]) and $L(y)$ $=L_{1}\left(L_{2}(y)\right)$ where $L_{2}(y)$ has $y_{1}, \cdots, y_{r}$ as a fundamental system of zeros and the group of $C\left\langle y_{1}, \cdots, y_{r}\right\rangle$ over $\mathcal{G}$ is the full linear group. Hence $L(y)$ $=L_{1}\left(y^{(r)}+t_{1} y^{(r-1)}+\cdots+t_{r}(y)\right.$ where $L_{1}(y) \in g\{y\}$. Let $L_{1}(y)=y^{(s)}+R_{1} y^{(s-1)}$ $+\cdots+R_{s} y \in \mathcal{G}\{y\}$ comparing coefficients in $L(y)=L_{1}\left(L_{2}(y)\right)$, we get

$$
\begin{aligned}
t_{r+1} & =P_{1}=t_{1}+R_{1}, \\
t_{r+2} & =P_{2}=s t_{1}^{\prime}+t_{2}+R_{1} t_{1}+R_{2}, \\
t_{r+i}=P_{i} & =\sum_{k=0}^{i-1} R_{k} \sum_{j=1}^{i-k}\left(\begin{array}{c}
s-k \\
i-k-j
\end{array}\right) t_{j}^{(i-k-j)}+R_{i} \quad(i=1, \cdots, s) .
\end{aligned}
$$

where

$$
\left(\begin{array}{c}
s-k \\
i-k-j
\end{array}\right)
$$

are the binomial coefficients and $R_{0}=1$.

We see that the $R_{i}(i=1, \cdots, s)$ are differential polynomials in $t_{1}, \cdots, t_{n}$ with coefficients in $C$. Also, $P_{1}, \cdots, P_{n}$ are differential polynomials in $R_{1}, \cdots, R_{s}, t_{1}, \cdots, t_{r}$ so that $C\left\langle P_{1}, \cdots, P_{n}\right\rangle \subset C\left\langle t_{1}, \cdots, t_{n}\right\rangle$. Hence $\mathcal{S}=C\left\langle t_{1}, \cdots, t_{n}\right\rangle$.

Set $L(t, y)=L_{1}\left(t, L_{2}(t, y)\right)$ where

$$
L_{2}(t, y)=y^{(r)}+t_{1} y^{(r-1)}+\cdots+t_{r} y
$$

and 


$$
L_{1}(t, y)=y^{(s)}+R_{1}\left(t_{1}, \cdots, t_{n}\right) y^{(s-1)}+\cdots+R_{s}\left(t_{1}, \cdots, t_{n}\right) y
$$

then

$$
L(t, y)=y^{(n)}+Q_{1}\left(t_{1}, \cdots, t_{n}\right) y^{(n-1)}+\cdots+Q_{n}\left(t_{1}, \cdots, t_{n}\right) y
$$

where

$$
Q_{i} \in C\left\{t_{1}, \cdots, t_{n}\right\} \quad(i=1, \cdots, n) .
$$

Let

$$
\left(t_{1}, \cdots, t_{n}, y_{1}, \cdots, y_{n}\right) \rightarrow\left(\bar{t}_{1}, \cdots, \bar{t}_{n}, \bar{y}_{1}, \cdots, \bar{y}_{n}\right)
$$

be any specialization over $C$ such that $\left(\bar{y}_{1}, \cdots, \tilde{y}_{n}\right)$ is a fundamental system of zeros of $L(\bar{t}, y)$ and $C\left\langle\bar{t}_{1}, \cdots, \bar{t}_{n}, \bar{y}_{1}, \cdots, \bar{y}_{n}\right\rangle$ is a P.V.E. of $C\left\langle\bar{t}_{1}, \cdots, \bar{t}_{n}\right\rangle$. Since $L(\bar{t}, y)=L_{1}\left(\bar{t}, L_{2}(\bar{t}, y)\right)$, any element $\left(a_{i j}\right)$ of the group $H$ of $C\left\langle\bar{t}_{1}, \cdots, \bar{t}_{n}\right.$, $\left.\bar{y}_{1}, \cdots, \bar{y}_{n}\right\rangle$ over $C\left\langle\bar{t}_{1}, \cdots, \bar{t}_{n}\right\rangle$ must take the subspace generated by $\bar{y}_{1}, \cdots, \bar{y}_{r}$ into itself so that $a_{r+k, m}=0(k, m=1, \cdots, s)$ so that $H$ is a subgroup of $G$. Furthermore since $r<n$ every zero of $W_{0}\left(y_{1}, \cdots, y_{r}\right)$ is a zero of $W_{0}\left(y_{1}, \cdots, y_{n}\right)$, so that every zero of $W_{0}\left(y_{1}, \cdots, y_{n}\right) W_{0}\left(y_{1}, \cdots, y_{r}\right)$ is a zero of $W_{0}\left(y_{1}, \cdots, y_{n}\right)$. Therefore $W_{0}\left(y_{1}, \cdots, y_{n}\right) \in\left\{W_{0}\left(y_{1}, \cdots, y_{n}\right)\right.$ - $\left.W_{0}\left(y, \cdots, y_{r}\right)\right\}$ (Ritt [3, p. 27]). Hence the conditions of Lemma 2 are satisfied and $L(t, y)=0$ is a "generic equation with group $G$."

EXAMPLE 1. Let $n=4$ and let $G$ be the group of all nonsingular matrices $\left(a_{i j}\right)$ with $a_{31}=a_{32}=a_{41}=a_{42}=0$ then

$$
\begin{aligned}
L(t, y)= & y^{(4)}+t_{3} y^{(3)}+t_{4} y^{(2)} \\
& +\left[t_{1}^{\prime \prime}+t_{3}\left(t_{1}^{\prime}+t_{2}-t_{1}^{2}\right)-3 t_{1} t_{1}^{\prime}-2 t_{1} t_{2}+t_{1} t_{4}+t_{1}^{3}+2 t_{2}^{\prime}\right] y^{\prime} \\
& +\left[t_{2}^{\prime \prime}+t_{3}\left(t_{2}^{\prime}-t_{1} t_{2}\right)+t_{4} t_{2}-t_{1} t_{2}^{\prime}-t_{2}^{2}+t_{1} t_{2}-2 t_{1}^{\prime} t_{2}\right] y .
\end{aligned}
$$

Of particular interest is a generic equation for the full triangular group. By iterating the result for the reducible group we find that the differential field $\mathcal{G}$ of invariants in $C\left\langle y_{1}, \cdots, y_{n}\right\rangle$ of the full triangular group is $C\left\langle t_{1}, \cdots, t_{n}\right\rangle$ where

$$
t_{i}=-\frac{W_{0}^{\prime}\left(y_{1}, \cdots, y_{i}\right)}{W_{0}\left(y_{1}, \cdots, y_{i}\right)} \quad(i=1, \cdots, n)
$$

For $n=2$,

$$
L(t, y)=y^{\prime \prime}+t_{2} y^{\prime}-\left(t_{2} t_{1}+t_{1}^{2}+t_{1}^{\prime}\right) y .
$$

For $n=3$,

$$
\begin{aligned}
L(t, y)= & y^{\prime \prime \prime}+t_{3} y^{\prime \prime}+\left(t_{1} t_{2}-t_{3} t_{2}-t_{2}^{2}-t_{1}^{\prime}-t_{1}^{2}\right) y^{\prime} \\
& +\left[t_{3}\left(t_{1} t_{2}-t_{1}^{2}-t_{1}^{\prime}\right)-t_{1}^{2} t_{2}+t_{1} t_{2}^{\prime}+t_{1} t_{2}^{2}-t_{1}^{\prime \prime}-2 t_{1} t_{1}^{\prime}\right] y .
\end{aligned}
$$




\section{The orthogonal and proper orthogonal group.}

TheOREm 2. Let $G$ be the orthogonal group of order $n$. Then the differential field $G$ of invariants of $G$ in $C\left\langle y_{1}, \cdots, y_{n}\right\rangle$ is purely differentially transcendental over $C$ and $G=C\left\langle t_{0}, \cdots, t_{n-1}\right\rangle$ where

$$
t_{m}=\sum_{k=1}^{n}\left(y_{k}^{(m)}\right)^{2} \quad(m=0,1,2, \cdots) .
$$

Proof. We show that

$$
2 \sum_{k=1}^{n} y_{k}^{(m)} y_{k}^{(m+i)}=\sum_{j=0}^{[i / 2]} a_{i j} t_{m+j}^{(i-2 j)} \quad(0 \leqq m<\infty, 1 \leqq i<\infty)
$$

where $[i / 2]$ denotes the greatest integer $\leqq i / 2$, and

$$
a_{i j}=(-1)^{j} \frac{i}{i-j}\left(\begin{array}{c}
i-j \\
j
\end{array}\right) \quad(1 \leqq i<\infty, 0 \leqq j \leqq[i / 2]) .
$$

Indeed, since $\sum_{k=1}^{n}\left(y_{k}^{(m)}\right)^{2}=t_{m}$ we have $2 \sum_{k=1}^{n} y_{k}^{(m)} y_{k}^{(m+1)}=t_{m}^{\prime}$ so that (1) holds for $0 \leqq m<\infty, i=1$. Differentiating this equation we obtain $2 \sum y_{k}^{(m)} y_{k}^{(m+2)}$ $=t_{m}^{\prime \prime}-2 t_{m+1}$ so that (1) also holds for $i=2$. Now let $i>2$ and suppose that (1) holds for lowest values of $i$ and for all $m$; differentiating (1) with $i$ replaced by $i-1$ we find

$$
\begin{aligned}
2 \sum_{k=1}^{n} y_{k}^{(m)} y_{k}^{(m+i)}= & \sum_{j=0}^{[(i-1) / 2]} a_{i-1, j} t_{m+j}^{(i-2 j)}-2 \sum_{k=1}^{n} y_{k}^{(m+1)} y_{k}^{(m+i-1)} \\
= & \sum_{j=0}^{[(i-1) / 2]} a_{i-1, j} t_{m+j}^{(i-2 j)}-\sum_{h=0}^{[(i-2) / 2]} a_{i-2, h} t_{m+1+h}^{(i-2-2 h)} \\
= & \sum_{j=0}^{[(i-1) / 2]} a_{i-1, i} t_{m+j}^{(i-2 j)}-\sum_{j=1}^{[i / 2]} a_{i-2, j-1} t_{m+j}^{(i-2 j)} \\
= & a_{i-1,0} t_{m}^{(i)}+\sum_{j=1}^{[(i-1) / 2]}\left(a_{i-1, i}-a_{i-2, j-1}\right) t_{m+j}^{(i-2 j)} \\
& -\left(\left[\frac{i}{2}\right]-\left[\frac{i-1}{2}\right]\right) a_{i-2,[i / 2]-1} t_{m+[i / 2]} \\
= & a_{i, 0} t_{m}^{(i)}+\sum_{j=1}^{[(i-1) / 2]} a_{i j} t_{m+j}^{(i-2 j)}+\left(\left[\frac{i}{2}\right]-\left[\frac{i-1}{2}\right]\right) a_{i,[i / 2]} t_{m+[i / 2]} \\
= & \sum_{j=0}^{[i / 2]} a_{i j}^{(i-2 j} t_{m+j}^{(i)}
\end{aligned}
$$

so that (1) holds for all $i \geqq 1$ and all $m \geqq 0$. This shows that $\sum_{k=1}^{n} y_{k}^{(m)} y_{k}^{(m+\imath)}$ $\in C\left\{t_{0}, t_{1}, \cdots, t_{n-1}\right\}$ whenever 


$$
2 m+i \leqq 2 n-2 \text { ( } i \text { even), } 2 m+i \leqq 2 n-1 \text { ( } i \text { odd). }
$$

In particular, setting $i=n-m$, we find that

$$
\sum_{k=1}^{n} y_{k}^{(m)} y_{k}^{(n)} \in C\left\{t_{0}, t_{1}, \cdots, t_{n-1}\right\} \quad(0 \leqq m \leqq n-1),
$$

for if $m<n-1$ then $2 m+n-m \leqq 2 n-2$ and if $m=n-1$ then $n-m$ is odd and $2 m+n-m=2 n-1$. But

$$
y_{k}^{(n)}=-\sum_{r=1}^{n} P_{r}\left(y_{1}, \cdots, y_{n}\right) y_{k}^{(n-r)}
$$

so that

$$
\sum_{r=1}^{n} P_{r}\left(y_{1}, \cdots, y_{n}\right) \sum_{k=1}^{n} y_{k}^{(m)} y_{k}^{(n-r)} \in C\left\{t_{0}, t_{1}, \cdots, t_{n-1}\right\} \quad(0 \leqq m \leqq n-1) .
$$

This gives rise to $n$ linear equations in $P_{1}, \cdots, P_{n}$ with coefficients in $C\left\{t_{0}, t_{1}, \cdots, t_{n-1}\right\}:$ moreover

$$
\operatorname{det}\left(\sum_{k=1}^{n} y_{k}^{(m)} y_{k}^{(n-r)}\right)=W_{0}^{2}\left(y_{1}, \cdots, y_{n}\right) \neq 0 .
$$

Hence $C\left\langle P_{1}\left(y_{1}, \cdots, y_{n}\right), \cdots, P_{n}\left(y_{1}, \cdots, y_{n}\right)\right\rangle \subset C\left\langle t_{0}, \cdots, t_{n-1}\right\rangle$. Since $t_{i}(i=0,1, \cdots, n-1)$ is left invariant by the orthogonal group and by no other nonsingular linear transformation, $\mathcal{G}=C\left\langle t_{0}, t_{1}, \cdots, t_{n-1}\right\rangle$.

COROLlaRY. Let $G$ be the proper orthogonal group of order $n$. Then the differential field $\mathrm{G}$ of invariants of $G$ in $C\left\langle y_{1}, \cdots, y_{n}\right\rangle$ is purely differentially transcendental over $C$.

Proof. Obviously $G=C\left\langle t_{0}, \cdots, t_{n-1}, W_{0}\left(y_{1}, \cdots, y_{n}\right)\right\rangle$. From (2) if we express $\left|\left(\sum_{k=1}^{n} y_{k}^{(m)} y_{k}^{(n-r)}\right)\right|$ as a differential polynomial in $t_{0}, \cdots, t_{n-1}$, the differential polynomial will contain $t_{n-1}$ only when $m=n-1$ and $r=1$. Hence we may solve (2) for $t_{n-1}$, so that

$$
\mathcal{S}=C\left\langle t_{0}, t_{1}, \cdots, t_{n-2}, W_{0}\left(y_{1}, \cdots, y_{n}\right)\right\rangle .
$$

\section{The symplectic group.}

THEOREM 3. Let $n$ be an even integer $>0$ and let $G$ be the symplectic group of order $n$ (i.e. the $n \times n$ algebraic matric group which leaves invariant the bilinear form $\left.\sum_{s=1}^{n / 2}\left(\mu_{2 s-1} \nu_{2 s}-\mu_{2 s} \nu_{2 s-1}\right)\right)$. Then the differential field $\mathcal{G}$ of invariants in $C\left\langle y_{1}, \cdots, y_{n}\right\rangle$ of $G$ is purely differentially transcendental over $C$ and $\mathrm{G}$ $=C\left\langle t_{0}, t_{1}, \cdots, t_{n-1}\right\rangle$ where

$$
t_{m}=\sum_{s=1}^{n / 2}\left(y_{2 s-1}^{(m)} y_{2 s}^{(m+1)}-y_{2 s-1}^{(m+1)} y_{2 s}^{(m)}\right) \quad(m=0,1,2, \cdots) .
$$


Proof. Define

$$
t_{i k}=\sum_{s=1}^{n / 2}\left(y_{2 s-1}^{(i)} y_{2 s}^{(i+k)}-y_{2 s-1}^{(i+k)} y_{2 s}^{(i)}\right)
$$

then

$$
t_{i}=t_{i 1}, \quad t_{i k}^{\prime}=t_{i+1, k-1}+t_{i, k+1} .
$$

We shall prove that

$$
t_{i k}=\sum_{j=1}^{[(k+1) / 2]} a_{k, j} t_{i+j-1}^{(k-2 j+1)}
$$

where

$$
a_{k, j}=(-1)^{j-1}\left(\begin{array}{l}
k-j \\
j-1
\end{array}\right) .
$$

(3) certainly holds for all $i \geqq 0, k=1,2$. Assume inductively that (3) holds for all $i \geqq 0$ and $1 \leqq k \leqq r$. Now,

$$
\begin{aligned}
t_{i, r+1}= & t_{i r}^{\prime}-t_{i+1, r-1}=\sum_{j=1}^{[(r+1) / 2]} a_{r j} t_{i+j-1}^{(r-2 j+2)}-\sum_{j=1}^{[r / 2]} a_{r-1, j} t_{1+j}^{(r-2 j)} \\
= & t_{i}^{(r)}+\sum_{j=2}^{[(r+1) / 2]}\left(a_{r j}-a_{r-1, j-1}\right) t_{i+j-1}^{(r-2 j+2)} \\
& -\left(\left[\frac{r}{2}\right]+1-\left[\frac{r+1}{2}\right]\right) a_{r-1,[r / 2]} t_{i+[r / 2]}^{(r-2[r / 2])} \\
= & \sum_{j=1}^{[(r+2) / 2]} a_{r+1, j} t_{i+j-1}^{(r+1-2 j+1)}
\end{aligned}
$$

which proves (3) for $k=r+1$, it therefore holds for all $1 \leqq k<\infty$. It follows from (3) that $t_{i k} \in C\left\langle t_{0}, t_{1}, \cdots, t_{n-1}\right\rangle$ whenever $2 i+k \leqq 2 n-1$. In particular $t_{i, n-i} \in C\left\langle t_{0}, \cdots, t_{n-1}\right\rangle(i=0,1, \cdots, n-1)$. Since

$$
y_{j}^{(n)}=-\sum_{r=1}^{n} P_{r}\left(y_{1}, \cdots, y_{n}\right) y^{(n-r)} \quad(j=1, \cdots, n)
$$

we have

$$
\begin{aligned}
t_{i, n-i} & =-\sum_{r=1}^{n} P_{r} \sum_{s=1}^{n / 2}\left(y_{2 s-1}^{(i)} y_{2 s}^{(n-r)}-y_{2 s}^{(i)} y_{2 s-1}^{(n-r)}\right) \\
& =-\sum_{r=1}^{n} P_{r} t_{i, n-r-i} \in C\left\langle t_{0}, t_{1}, \cdots, t_{n-1}\right\rangle
\end{aligned}
$$

where $t_{i, n-k-i}=-t_{n-k, i-(n-k)}$ if $n-k<i$, we thus obtain a system of $n$ linear 
equations in $P_{1}, \cdots, P_{n}$ with coefficients $\in C\left\langle t_{0}, t_{1}, \cdots, t_{n-1}\right\rangle$. If we define integers $\alpha_{\mu \nu}$ by the equation

$$
\sum_{s=1}^{n / 2}\left(y_{2 s-1} y_{2 s}^{\prime}-y_{2 s} y_{2 s-1}^{\prime}\right)=\sum \alpha_{\mu \nu} y_{\mu} y_{\nu}^{\prime}
$$

then the det of the linear system

$$
\begin{aligned}
& =\operatorname{det}\left(\sum y_{2 s-1}^{(i)} y_{2 s}^{(n-r)}-y_{2 s}^{(i)} y_{2 s-1}^{(n-r)}\right)=\operatorname{det}\left(\sum_{\mu, \nu} \alpha_{\mu \nu} y_{\mu}{ }^{(i)} y_{\nu}{ }^{(n-r)}\right) \\
& =\operatorname{det}\left(y_{\mu}^{(i)}\right) \cdot \operatorname{det}\left(\alpha_{\mu \nu}\right) \cdot \operatorname{det}\left(y_{\nu}^{(n-r)}\right)=\operatorname{det}\left(\alpha_{\mu \nu}\right) W_{0}^{2}\left(y_{1}, \cdots, y_{n}\right) .
\end{aligned}
$$

Since det $\left(\alpha_{\mu \nu}\right)=1$ we have

$$
\operatorname{det}\left(\sum_{s=1}^{n / 2} y_{2 s-1}^{(i)} y_{2 s}^{(n-r)}-y_{2 s}^{(i)} y_{2 s-1}^{(n-r)}\right)=W_{0}^{2}\left(y_{1}, \cdots, y_{n}\right) \neq 0 .
$$

It follows that the linear system may be solved for $P_{1}, \cdots, P_{n}$, so that $C\left\langle P_{1}, \cdots, P_{n}\right\rangle \subset C\left\langle t_{0}, t_{1}, \cdots, t_{n-1}\right\rangle$. Since $C\left\langle t_{0}, t_{1}, \cdots, t_{n-1}\right\rangle$ is left invariant by $G$ and by no other nonsingular linear transformation, $C\left\langle t_{0}, t_{1}, \cdots, t_{n-1}\right\rangle$ $=g$.

8. Generic equations for the orthogonal and the symplectic group.

LEMMA 3. Let $\mathcal{F}\left\langle\omega_{1}, \cdots, \omega_{n}\right\rangle$ be any differential field with field of constants $C$. Let $\left(\omega_{1}, \cdots, \omega_{n}\right)$ be a solution of either one of the following sets of equations:

$$
\begin{array}{r}
\sum_{i, j} a_{i j} y_{i}^{(\mu)} y_{j}^{(\mu)}=0 \quad(i, j=1, \cdots, n, \mu=0,1, \cdots, n-1) \\
a_{i j}=a_{j i} \in C \operatorname{rank}\left(a_{i j}\right)>0 . \\
\sum_{i, j} b_{i j} y_{i}^{(\mu)} y_{j}^{(\mu+1)}=0 \quad(i, j=1, \cdots, n, \mu=0,1, \cdots, n-1) \\
b_{i j}=-b_{j i} \in C \operatorname{rank}\left(b_{i j}\right)>0 .
\end{array}
$$

Then $\omega_{1}, \cdots, \omega_{n}$ are linearly dependent.

Proof. Assume the theorem to be false then $\omega_{1}, \cdots, \omega_{n}$ are linearly independent. Let rank of $\left(a_{i j}\right),\left(b_{i j}\right)$ be $\nu>0$; then there exists a nonsingular linear transformation $S$ such that $S \omega_{k}=\pi_{k}$ and $\left(\pi_{1}, \cdots, \pi_{\nu}\right)$ is a solution of

$$
\sum_{k=1}^{\nu}\left(y_{k}^{(\mu)}\right)^{2}=0 \quad(\mu=0,1, \cdots, \nu-1) \text { if }\left(\omega_{1}, \cdots, \omega_{n}\right)
$$

is a solution of (B). Similarly, there exists $S$ such that $S \omega_{k}=\pi_{k}$ and $\left(\pi_{1}, \cdots, \pi_{\nu}\right)$ is a solution of 


$$
\sum_{s=1}^{\nu / 2}\left(y_{2 s-1}^{(\mu)} y_{2 s}^{(\mu+1)}-y_{2 s}^{(\mu)} y_{2 s-1}^{(\mu+1)}\right)=0 \quad(\mu=0,1, \cdots, \nu-1)
$$

if $\left(\omega_{1}, \cdots, \omega_{n}\right)$ is a solution of (C). Now, from (1) and (2) we see that $W_{0}\left(y_{1}, \cdots, y_{v}\right)$ belongs to the ideal $\left\{\sum_{k=1}^{\nu} y_{k}^{2}, \sum_{k=1}^{v} y_{k}^{\prime 2}, \cdots, \sum_{k=1}^{v}\left(y_{k}^{(\nu-1)^{2}}\right\}\right.$. Similarly, from (3) and (4) we see that $W_{0}\left(y_{1}, \cdots, y_{v}\right)$ belongs to the ideal

$$
\left\{\sum_{s=1}^{\nu / 2}\left(y_{2 s-1} y_{2 s}^{\prime}-y_{2 s} y_{2 s-1}^{\prime}\right), \cdots, \sum_{s=1}^{\nu / 2}\left(y_{2 s-1}^{(\nu-1)} y_{2 s}^{(\nu)}-y_{2 s}^{(\nu-1)} y_{2 s-1}^{(\nu)}\right)\right\} \text {. }
$$

In either case $W_{0}\left(\pi_{1}, \cdots, \pi_{v}\right)=0$ contradicting our assumption that $\omega_{1}, \cdots, \omega_{n}$ are linearly independent. Hence $\omega_{1}, \cdots, \omega_{n}$ are linearly dependent.

THEOREM 4. Let $G$ be either the orthogonal group of order $n$ over $C$, or else the symplectic group of even order $n$ over $C$. Express the differential polynomials $P_{i}\left(y_{1}, \cdots, y_{n}\right)$ in the form

$$
\begin{aligned}
P_{i}\left(y_{1}, \cdots, y_{n}\right) & =\frac{Q_{i}\left(t_{0}, t_{1}, \cdots, t_{n-1}\right)}{Q_{0}\left(t_{0}, t_{1}, \cdots, t_{n-1}\right)} \quad(i=1, \cdots, n), \\
Q_{i}\left(t_{0}, t_{1}, \cdots, t_{n-1}\right) & \in C\left\{t_{0}, t_{1}, \cdots, t_{n-1}\right\} \quad(i=0,1, \cdots, n)
\end{aligned}
$$

where

$$
t_{j}=\sum_{k=1}^{n}\left(y_{k}^{(j)}\right)^{2}
$$

or

$$
t_{j}=\sum_{s=1}^{v / 2}\left(y_{2 s-1}^{(j)} y_{2 s}^{(j+1)}-y_{2 s}^{(j)} y_{2 s-1}^{(j+1)}\right)
$$

according as $G$ is orthogonal or symplectic. Then

$$
L(t, y)=Q_{0}\left(t_{0}, \cdots, t_{n-1}\right) y^{(n)}+Q_{1}\left(t_{0}, \cdots, t_{n-1}\right) y^{(n-1)}+\cdots+Q_{n} y=0
$$

is a "generic equation with group $G$."

Proof. We shall give the proof for the orthogonal case. The proof for the symplectic case is entirely similar.

Let

$$
\left(t_{0}, t_{1}, \cdots, t_{n-1}, y_{1}, \cdots, y_{n}\right) \rightarrow\left(\bar{t}_{0}, \bar{t}_{1}, \cdots, \bar{t}_{n-1}, \omega_{1}, \cdots, \omega_{n}\right)
$$

be a specialization over $C$ such that $\left(\omega_{1}, \cdots, \omega_{n}\right)$ is a fundamental system of zeros of $L(\bar{t}, y)$ and $C\left\langle\bar{t}_{0}, \bar{t}_{1}, \cdots, \bar{t}_{n-1}, \omega_{1}, \cdots, \omega_{n}\right\rangle$ is a P.V.E. of $C\left\langle\bar{t}_{0}, \bar{t}_{1}, \cdots\right.$, $\left.\bar{t}_{n-1}\right\rangle$ with group $H$. Let $\sigma \in H$; then

$$
\sum_{k=1}^{n}\left(\omega_{k}^{(i)}\right)^{2}=\bar{t}_{i}=\sigma \bar{t}_{i}=\sum_{m, p} a_{m p} \omega_{m}^{(i)} \omega_{p}^{(i)}
$$


where

$$
a_{m p}=a_{p m} \in C,
$$

so that

$$
\sum_{m, p} a_{m p} \omega_{m}^{(i)} \omega_{p}^{(i)}-\sum\left(\omega_{k}^{(i)}\right)^{2}=\sum_{m, p} b_{m p} \omega_{m}^{(i)} \omega_{p}^{(i)}=0
$$

where

$$
b_{m p}= \begin{cases}a_{m p} & \text { if } m \neq p \\ a_{m p}-1 & \text { if } m=p .\end{cases}
$$

Since $b_{m p}=b_{p m}$, by Lemma 3 if rank of $\left(b_{m p}\right)$ is not zero, $\omega_{1}, \cdots, \omega_{n}$ are linearly dependent contrary to our hypothesis. Hence rank of $\left(b_{m p}\right)$ is zero and

$$
a_{m p}=\left\{\begin{array}{l}
0 \text { if } m \neq p \\
1 \text { if } m=p
\end{array}\right.
$$

so that $\sigma$ belongs to the orthogonal group. Hence $H \subseteq G$.

It follows from (1) and (2) that the $t_{i}(i=0,1, \cdots, n-1)$ are differential polynomials in $y_{1}, \cdots, y_{n}$ and that

$$
Q_{0}\left(t_{0}, t_{1}, \cdots, t_{n-1}\right)=(-2)^{n} W_{0}^{2}\left(y_{1}, \cdots, y_{n}\right)
$$

so that the conditions of Lemma 2 are satisfied and therefore

$$
L(t, y)=Q_{0}\left(t_{0}, t_{1}, \cdots, t_{n-1}\right) y^{(n)}+\cdots+Q_{n}\left(t_{0}, \cdots, t_{n-1}\right) y=0
$$

is a "generic equation with group $G$."

EXAMPLE 1. Let $G$ be the $2 \times 2$ orthogonal group then

$$
\left(t_{0}^{\prime 2}-4 t_{0} t_{1}\right) y^{\prime \prime}+\left[2\left(t_{0} t_{1}\right)^{\prime}-t_{0} t_{0}^{\prime \prime}\right] y^{\prime}+\left(2 t_{0}^{\prime \prime} t_{1}-t_{0}^{\prime} t_{1}^{\prime}-4 t_{1}^{2}\right) y=0
$$

is a "generic equation with group $G . "$

EXAMPLE 2 . Let $G$ be the $3 \times 3$ orthogonal group then

$$
L(t, y)=Q_{0} y^{\prime \prime \prime}+Q_{1} y^{\prime \prime}+Q_{2} y^{\prime}+Q_{3} y=0
$$

where

$$
\begin{aligned}
Q_{0}= & 2\left\{t_{2}\left(t_{0}^{\prime 2}-4 t_{0} t_{1}\right)-t_{1}^{\prime}\left[t_{0}^{\prime}\left(t_{0}^{\prime \prime}-2 t_{1}\right)-2 t_{0} t_{1}^{\prime}\right]+t_{1}\left(t_{0}^{\prime \prime}-2 t_{1}\right)^{2}\right\}, \\
Q_{1}= & \left(3 t_{1}^{\prime}-t_{0}^{\prime \prime \prime}\right)\left[2 t_{1}\left(t_{0}^{\prime \prime}-2 t_{1}\right)-t_{0}^{\prime} t_{2}^{\prime}\right]+\left(t_{1}^{\prime \prime}-2 t_{2}\right)\left[t_{0}^{\prime}\left(t_{0}^{\prime \prime}-2 t_{1}\right)-2 t_{0} t_{1}^{\prime}\right] \\
& -t_{2}^{\prime}\left(t_{0}^{\prime 2}-4 t_{0} t_{1}\right) \\
\text { (6) } Q_{2}= & \left(t_{0}^{\prime \prime}-2 t_{1}\right)\left[\left(2 t_{2}-t_{1}^{\prime \prime}\right)\left(t_{0}^{\prime \prime}-2 t_{1}\right)-t_{1}^{\prime}\left(3 t_{1}^{\prime}-t_{0}^{\prime \prime \prime}\right)+t_{0}^{\prime} t_{2}^{\prime}\right] \\
& +2 t_{2}\left[\left(3 t_{1}^{\prime}-t_{0}^{\prime \prime \prime}\right) t_{0}^{\prime}-\left(2 t_{2}-t_{1}^{\prime \prime}\right) 2 t_{0}\right]-2 t_{0} t_{1}^{\prime} t_{2}^{\prime}, \\
Q_{3}= & \left(3 t_{1}^{\prime}-t_{0}^{\prime}\right)\left(t_{1}^{\prime 2}-4 t_{1} t_{2}\right)+\left(t_{0}^{\prime \prime}-2 t_{1}\right)\left[\left(t_{1}^{\prime \prime}-2 t_{2}\right) t_{1}^{\prime}-2 t_{1} t_{2}^{\prime}\right] \\
& +2 t_{0}^{\prime} t_{2}\left(2 t_{2}-t_{1}^{\prime \prime}\right)+t_{0}^{\prime} t_{1}^{\prime} t_{2}^{\prime}
\end{aligned}
$$


is a "generic equation with group $G . "$

Let $G$ be the $3 \times 3$ proper orthogonal group then by the corollary of Theorem 2 the differential subfield of $C\left\langle y_{1}, y_{2}, y_{3}\right\rangle$ which is left invariant by $G$ is $C\left\langle t_{0}, t_{1}, W_{0}\left(y_{1}, \cdots, y_{n}\right)\right\rangle$ where $t_{0}, t_{1}$ is the same as for the orthogonal case. We may solve for $t_{2}$ from (6) recalling that $Q_{0}=-8 W_{0}^{2}\left(y_{1}, y_{2}, y_{3}\right)$ we obtain

$$
t_{2}=\frac{-4 W_{0}+t_{1}^{\prime}\left[t_{0}^{\prime}\left(t_{0}^{\prime \prime}-2 t_{1}\right)-2 t_{0} t_{1}^{\prime}\right]-t_{1}\left(t_{0}^{\prime \prime}-2 t_{1}\right)^{2}}{t_{0}^{\prime 2}-4 t_{0} t_{1}}
$$

if we substitute this expression for $t_{2}$ in $Q_{2}, Q_{3},\left(Q_{1}=8 W_{0} W_{0}^{\prime}\right)$ we obtain

$$
L(t, y)=y^{\prime \prime \prime}-\frac{W_{0}^{\prime}}{W_{0}} y^{\prime \prime}+R_{1}\left(t_{0}, t_{1}, W_{0}\right) y^{\prime}+R_{2}\left(t_{0}, t_{1}, W_{0}\right) y
$$

where $R_{1}, R_{2} \in C\left\langle t_{0}, t_{1}, W_{0}\right\rangle$. The following example shows that (8) is not a "generic equation with group $G$ " where $G$ is the proper orthogonal group.

Example 3. Let $F=C\langle x\rangle$ where $C$ is the complex numbers and $x^{\prime}=1$. Let

$$
L(y)=y^{\prime \prime \prime}+2 x y^{\prime}+y
$$

and let $\left(t_{0}, t_{1}, t_{2}\right) \rightarrow(0,1,2 x)$ be the specialization over $C$. Then from (6) we have $\bar{Q}_{0}=8, \bar{Q}_{1}=0, \bar{Q}_{2}=16 x, \bar{Q}_{3}=8$ so that $(5)$ becomes $L(\bar{t}, y)=8\left(y^{\prime \prime \prime}+2 x y^{\prime}+y\right)$. It can be shown that this specialization can be extended to a specialization $\left(t_{0}, t_{1}, t_{2}, y_{1}, y_{2}, y_{3}\right) \rightarrow\left(0,1,2 x, \omega_{1}, \omega_{2}, \omega_{3}\right)$ over $\mathcal{F}$ where $\mathcal{F}\left\langle\omega_{1}, \omega_{2}, \omega_{3}\right\rangle$ is a P.V.E. of $\mathcal{F}$. Hence the algebraic matric group $H$ of $\mathscr{F}\left\langle\omega_{1}, \omega_{2}, \omega_{3}\right\rangle$ over $\mathcal{F}$ must be a subgroup of the orthogonal group. Since the coefficient of $y^{\prime \prime}$ in $L(y)$ is 0 , $H$ is a subgroup of the unimodular group, so that $H$ is a subgroup of the proper orthogonal group. We are going to show that $H=$ proper orthogonal group.

For, let $H_{0}$ be the component of the identity of $H$ and let dimension of $H_{0} \leqq 2$ then $H_{0}$ is solvable (for the dimension of the Lie algebra corresponding to $H_{0}$ would have dimension $\leqq 2$ and is therefore solvable). Then there exists $\pi$ a zero of $L(y)$ such that $\pi^{i} \pi^{-1}$ is algebraic over $\mathcal{F}$, but the coefficients of $L(y)$ are regular in the whole complex plane so that $\pi^{\prime} \pi^{-1}$ can not have any branch points and must be a rational function of $x$. Now, $\pi^{\prime} \pi^{-1}$ is a zero of

$$
F(z)=z^{\prime \prime}+3 z z^{\prime}+z^{3}+2 x z+1
$$

if $\pi^{\prime} \pi^{-1}$ has a pole of order $r$ at a place $c \neq \infty$ then $r=1$ (for $z^{3}, z z^{\prime}, z^{\prime \prime}$ have poles of order $3 r, 2 r+1, r+2$ respectively; equating $3 r=2 r+1$ we get $r=1$ ). Let $u=x^{-1}$ then

$$
F(z)=u^{5} \ddot{z}-3 u^{3} z \dot{z}+2 u^{4} \dot{z}+u z^{3}+2 z+u
$$

where $\dot{z}, \ddot{z}$ denotes differentiation with respect to $u$. Let $r$ be the order of the pole of $\pi^{\prime} \pi^{-1}$ at $u=0$ then $u\left(\pi^{\prime} \pi^{-1}\right)^{3}$ has a pole of order $3 r-1$ which is greater than any other term in $F(z)$. Hence $\pi^{\prime} \pi^{-1}$ does not have a pole at $x=\infty$ so that 


$$
\pi^{\prime} \pi^{-1}=a_{0}+\sum_{i=1}^{n} a_{i}\left(x-c_{i}\right)^{-1} \quad a_{i j} c_{i} \in C .
$$

Solving for $\pi$ we get $\pi=d e^{a_{0} x} \prod_{i=1}^{n}\left(x-c_{i}\right)^{a_{i}}$. Since $\pi$ is regular in the whole plane the $a_{i}$ must be positive integers, so that $\pi=e^{a_{0} x} P(x), P(x)$ a polynomial. Substituting $\pi$ in $L(y)$ we find that $P(x)$ must be a zero of

$$
K(z)=z^{\prime \prime \prime}+3 a_{0} z^{\prime \prime}+\left(3 a_{0}^{2}+2 x\right) z^{\prime}+\left(a_{0}^{3}+2 a_{0} x+1\right) z .
$$

If $n$ is the degree of $P(x)$ then $2 a_{0} x z$ will have degree $n+1$ and all the other terms in $K(z)$ have lower degree. Hence $a_{0}=0$ and $\pi=P(x)$. Let $P(x)$ $=\sum_{i=0}^{n} c_{i} x^{i}$, then we must have $c_{n} x^{n}+2 x c_{n} x^{n-1}=0$ so that $c_{n}=0$. Hence $H_{0}$ is not solvable and dimension of $H>2$. But $H$ is a subgroup of the proper or thogonal group which has dimension 3 and is connected. Hence $H=$ proper orthogonal group.

Now, the specialization $t_{0} \rightarrow 0$ makes the denominator in (7) vanish, and it is easily checked that the denominators of $R_{1}\left(t_{0}, t_{1}, W_{0}\right)$ and $R_{2}\left(t_{0}, t_{1}, W_{0}\right)$ in (8) also vanish. Hence there does not exist a specialization $\left(t_{0}, t_{1}, W_{0}, y_{1}\right.$, $\left.y_{2}, y_{3}\right) \rightarrow\left(\bar{t}_{0}, \bar{t}_{1}, \bar{W}_{0}, \omega_{1}, \omega_{2}, \omega_{3}\right)$ over $\mathcal{F}$ such that $L(\bar{t}, y)=L(y)$, so that $L(t, y)$ of (8) is not a "generic equation with group G."

\section{REFERENCES}

1. E. R. Kolchin, Existence theorems connected with the Picard-Vessiot theory of homogeneous linear ordinary differential equations, Bull. Amer. Math. Soc. vol. 54 (1948) pp. 927-932.

2. - Algebraic matric groups and the Picard-Vessiot theory of homogeneous linear ordinary differential equations, Ann. of Math. vol. 49 (1948) pp. 1-42.

3. J. F. Ritt, Differential algebra, New York, Amer. Math. Soc. Colloquium Publications, vol. $33,1950$.

4. E. Noether, Gleichungen mit vorgeschriebener Gruppe, Math. Ann. vol. 78 pp. 221-229.

5. B. L. van der Waerden, Moderne Algebra, vol. 1, Berlin, Julius Springer, 1940.

Columbia University,

NEW YORK, N. Y. 